\title{
COMPOUND GROUP EXTENSIONS. I. CONTINUATIONS OF NORMAL HOMOMORPHISMS $\left({ }^{1}\right)$
}

\author{
BY \\ ROBERT L. TAYLOR
}

1. Introduction. A normal homomorphism is a homomorphism $\phi: K \rightarrow G$ of a group $K$ into a group $G$, such that $\phi(K)$ is a normal subgroup of $G$. We shall denote $\phi^{-1}(0)$ by $X, \phi(K)$ by $N$, and $G / N$ by $Q$; then the normal homomorphism can be displayed as an exact sequence

$$
0 \rightarrow X \rightarrow K \stackrel{\phi}{\rightarrow} G \stackrel{\mu}{\rightarrow} Q \rightarrow 0,
$$

where $\mu$ denotes the natural homomorphism. In fact one could consider, as the object of study, an arbitrary exact sequence of "length 4 ," as in (1.1); one would then need a symbol for the given isomorphism of $X$ into $K$. It will not matter, even notationally, whether or not the elements of $Q$ are actual cosets in $G$, so long as $\mu$ is a given homomorphism of $G$ onto $Q$ with kernel $N=\phi(K)$.

None of the groups involved is assumed to be abelian; but we adopt the following convention: all groups will be written additively, whether abelian or not, except the group $A(L)$ of all automorphisms of any group $L$, and subgroups of $A(L)$. Factor groups of $A(L)$ will be written additively. The "sum" of two functions with values in a group will mean the function obtained by adding values, or by multiplying values in the case of mappings into $A(L)$; the "product" of two functions will always mean their composition.

By a continuation of $\phi: K \rightarrow G$ we mean a group $E$, containing $K$ as a subgroup, together with a homomorphism $\bar{\phi}: E \rightarrow G$ such that the top row in the diagram

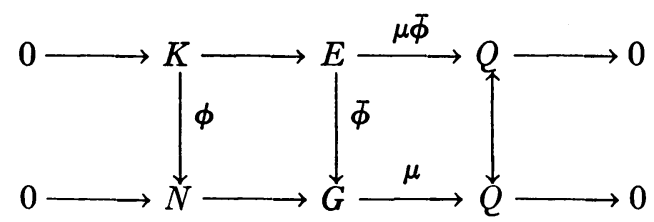

Presented to the Society, December 29, 1950, under the title The extendibility of a crossed module; received by the editors April 7, 1952 and, in revised form, August 19, 1952.

(1) This is the first of several papers devoted to an exposition of the contents of [13], combined with related results discovered subsequently. [13] was written under the supervision of Prof. J. H. C. Whitehead, to whom the author is indebted for many valuable suggestions. Some of the subsequent work included in this paper was partially supported by the Office of Scientific Research (ARDC). Numbers in brackets refer to the list of references at the end of the paper. 
is exact, and such that the left-hand rectangle is commutative $\left({ }^{2}\right)$. Unlabelled arrows represent identity injections, except those of the form $\rightarrow 0$.

Equivalently, one may demand that the bottom row be exact, and the right-hand rectangle commutative, in the diagram $\left({ }^{2}\right)$

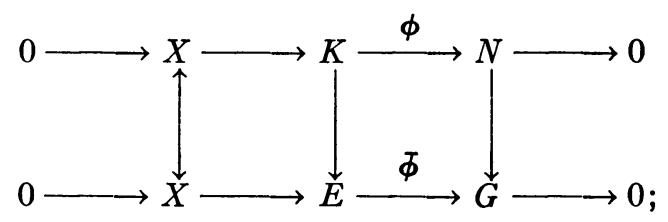

in either case, the conditions required of $\phi: E \rightarrow G$ are

$$
\bar{\phi} \mid K=\phi, \quad \bar{\phi}(E)=G, \quad \bar{\phi}^{-1}(0)=X .
$$

In the presence of (1.4a), condition (1.4c) is equivalent to each of the following:

$$
\bar{\phi}^{-1}(0) \subset X ; \quad \bar{\phi}^{-1}(N) \subset K ; \quad \bar{\phi}^{-1}(N)=K .
$$

Diagram (1.2) corresponds to regarding $E$ primarily as an extension of $Q$ by $K$, together with a homomorphism $\bar{\phi}: E \rightarrow G$ which induces a given homomorphism (namely $\phi$ ) of $K$ into $G$ and which also induces a given homomorphism (namely the identity mapping) of $Q=E / K$ into $Q=G / N$. In (1.3), we regard $E$ primarily as an extension of $G$ by $X$, which "continues" the given extension $K$ of $N$ by $X$. The point of view suggested by (1.2) will be generalized in [9], and underlies the approach used in the present paper; that suggested by (1.3) will be generalized in [1].

Two continuations $(E, \phi)$ and $\left(E^{\prime}, \Phi^{\prime}\right)$ of $\phi: K \rightarrow G$ will be called isomorphic if there exists an isomorphism $W: E^{\prime} \approx E$ such that

$$
W \mid K=\text { identity; } \quad \Phi W=\Phi^{\prime} .
$$

A continuation $(E, \Phi)$ of $\phi: K \rightarrow G$ determines in a natural way a homomorphism $\theta: G \rightarrow A_{X}(K) / c(X)$ of $G$ into the group $A_{X}(K) / c(X)$ of all automorphisms of $K$ which map $X$ onto itself, modulo those automorphisms which arise from conjugation of $K$ by elements of $X$. Any homomorphism $\theta: G$ $\rightarrow A_{X}(K) / c(X)$ satisfying appropriate commutativity conditions is called a modular structure on $\phi: K \rightarrow G$; the pair $(\phi, \theta)$ is called a pseudo-module. By an extension of the pseudo-module $(\phi, \theta)$ we mean a continuation of $\phi: K \rightarrow G$ which gives rise to the given modular structure $\theta$. The study of continuations of a normal homomorphism thus reduces to the study of extensions of a pseudomodule.

A pseudo-module in which $X \subset Z_{K}$, where $Z_{K}$ denotes the center of $K$,

(2) In (1.2), the right-hand rectangle is automatically commutative, and the bottom row is automatically exact. In (1.3), the left-hand rectangle is automatically commutative, and the top row is automatically exact. 
is the same thing as a crossed module, in the sense of J. H. C. Whitehead [14]. Our first main result is that the extension problem for pseudo-modules reduces to the extension problem for crossed modules, in the following way. With every pseudo-module we associate a certain crossed module, in a natural fashion; we then show that the extensions of a given pseudo-module are in a natural 1-1 correspondence with the extensions of the associated crossed module. In particular, a pseudo-module is extendible if and only if its associated crossed module is extendible.

The obstruction of a crossed module has been defined by MacLane and Whitehead [6;7]; it is an element of $H^{3}(Q, X)$, with $Q$ operating on $X$ in the manner induced by $\theta: Q \rightarrow A_{X}(K)$. We show:

(a) a crossed module is extendible if and only if its obstruction vanishes;

(b) $H^{2}(Q, X)$ operates in a natural way as a simply transitive transformation group on the set of all isomorphism-classes of extensions of the given crossed module.

Since the empty set is never regarded as an equivalence class, the set of all isomorphism-classes of extensions of a crossed module is nonempty if and only if the crossed module is extendible. However, since every group operates as a simply transitive transformation group on the empty set, result (b) holds whether the crossed module is extendible or not. In the extendible case, any choice of a particular isomorphism-class of extensions as "base point" induces a 1-1 correspondence between $H^{2}(Q, X)$ and the set of all isomorphismclasses of extensions.

Now let $(\phi, \theta)$ be a pseudo-module, and let $\left(\phi^{\prime}, \theta^{\prime}\right)$ be its associated crossed module. The construction is such that $K=K^{\prime}$ and $Q=Q^{\prime}$; however, $\phi^{\prime-1}(0)$ $=\phi^{-1}(0) \cap Z_{K}$. Hence, if we denote $\phi^{-1}(0)$ by $X$, the obstruction of $\left(\phi^{\prime}, \theta^{\prime}\right)$ is an element of $H^{3}\left(Q, X \cap Z_{K}\right)$, where the operation of $Q$ on $X \cap Z_{K}$ is induced by $\theta: G \rightarrow A_{X}(K) / c(X)$. We call this element of $H^{3}\left(Q, X \cap Z_{K}\right)$ the obstruction of $(\phi, \theta)$. Then results (a) and (b) automatically imply their own generalizations, as follows:

(c) a pseudo-module is extendible if and only if its obstruction vanishes;

(d) $H^{2}\left(Q, X \cap Z_{K}\right)$ operates in a natural way as a simply transitive transformation group on the set of all isomorphism-classes of extensions of the given pseudo-module.

These results are clearly exact analogues of the theorems of Eilenberg and MacLane [5] on group extensions with a nonabelian kernel ${ }^{(3)}$. In fact, a $Q$-kernel $\theta: Q \rightarrow A(K) / I(K)$ can be made into a pseudo-module in at least two different ways. On the one hand, MacLane has shown [6] that the concept of a $Q$-kernel is equivalent to that of a crossed module in which $X=Z_{K}$; in this construction, " $G$ " is a certain subgroup of the direct sum $Q+A(K)$, and " $\phi: K \rightarrow G$ " is the natural homomorphism of $K$ into $A(K)$ defined by conjugation, followed by the natural injection of $A(K)$ into $Q+A(K)$. On the other hand, if we define $G=Q, \phi(K)=0$, then $X=K$, and $(\phi, \theta)$ is a pseudo-module.

(3) See also $[2 ; 8]$. 
Whether we regard a $Q$-kernel as a crossed module in the manner of MacLane, or as a pseudo-module by taking $\phi=0$, the results on extensions of a $Q$-kernel follow immediately as special cases of the above. Moreover, if $\theta: Q$ $\rightarrow A(K) / I(K)$ is a $Q$-kernel, $G=Q, \phi=0$, and $\left(\phi^{\prime}, \theta^{\prime}\right)$ is the crossed module associated with the pseudo-module $(\phi, \theta)$ in the sense of this paper, then $\left(\phi^{\prime}, \theta^{\prime}\right)$ is the same as the crossed module constructed from $\theta: Q \rightarrow A(K) / I(K)$ by MacLane $\left.{ }^{4}\right)$.

The generalization from $Q$-kernels to pseudo-modules has been accompanied by a certain amount of reorganization. Since the results on group extensions are needed in the revised form for this and later work $[1 ; 10]$, they are summarized (as revised) in $\$ 5$.

In $\$ 14$, we return to the concept of the crossed module associated with a given pseudo-module, and prove that the concept of a pseudo-module is in fact equivalent to that of a crossed module together with a subgroup $Y$ of $K$ satisfying certain conditions; $Y$ becomes the $\phi^{-1}(0)$ of the corresponding pseudo-module.

If $G$ is a topological group, $N$ is the arcwise component of the identity, and $\phi: K \rightarrow N$ is a universal covering group of $N$, then $\phi: K \rightarrow G$ is a normal homomorphism which satisfies $X \subset Z_{K}$ and which admits one and only one modular structure $\theta: G \rightarrow A_{X}(K)$ such that $\theta(G)$ is contained in the group of all topological automorphisms of $K$. The extensions of the crossed module $(\phi, \theta)$ can be identified with the universal covering groups of $G$. Hence the results of this paper provide a classification of the universal covering groups of a nonconnected topological group whose arcwise components admit universal covering spaces; this leads to a classification of the non-universal covering groups as well [11]. In [12], it is shown that, for certain purposes, the local restrictions on $N$ involved in the assumption that $N$ admits a universal covering space can be eliminated.

The results on covering groups play a role in the classification of fiber bundles over a compact 2-manifold [13] and, in that connection, formed the original motivation for the present work.

2. Notation; some preliminary identities. If $P$ denotes a group, the symbols $A(P), I(P)$, and $Z_{P}$ will denote, respectively, the group of all automorphisms of $P$, the group of all inner automorphisms of $P$, and the center of $P . c_{P}: P \rightarrow I(P)$ will denote the natural homomorphism defined by

$$
\left(c_{P} p\right)\left(p^{\prime}\right)=p+p^{\prime}-p .
$$

If $M$ denotes a subgroup of $P, A_{M}(P)$ will denote the group of all automorphisms of $P$ which map $M$ onto itself. If $M$ is a normal subgroup of $P, C_{M}: P \rightarrow A(M)$ will denote the natural homomorphism defined by

$$
\left(C_{M} p\right)(m)=p+m-p .
$$

(4) This last assertion will become self-evident to the reader; it will not be discussed in the text. 
The subscripts will frequently be omitted from the symbols $c_{P}$ and $C_{M}$.

Throughout most of this paper, attention will be confined to a single fixed normal homomorphism (1.1). The letters introduced in this and the previous section to denote various groups and homomorphisms derived from (1.1) will be used in the same sense throughout.

The symbol $c_{K}(X)$ denotes the image of $X$ in $I(K)$ under the natural homomorphism $c_{K}: K \rightarrow I(K)$; it will generally be denoted $c(X)$, although it is a group of automorphisms of $K$, not of $X$. Both $c(X)$ and $I(K)$ are normal subgroups of $A_{X}(K)$, although $c(X)$ is not, in general, a normal subgroup of $A(K)$.

The following will denote the natural homomorphisms:

$$
\begin{aligned}
\lambda: A_{X}(K) & \rightarrow A_{X}(K) / c(X), \\
\omega: A_{X}(K) / c(X) & \rightarrow A_{X}(K) / I(K), \\
\nu: A_{X}(K) & \rightarrow A_{X}(K) / I(K), \\
\bar{\nu}: A(N) & \rightarrow A(N) / I(N), \\
\eta: A_{X}(K) & \rightarrow A\left(X \cap Z_{K}\right) .
\end{aligned}
$$

Clearly $\eta$ [which is defined by restricting each element of $A_{X}(K)$ to $\left.X \cap Z_{K}\right]$ maps $I(K)$, and a fortiori $c(X)$, to the identity element of $A\left(X \cap Z_{K}\right)$. Hence $\eta$ induces homomorphisms

$$
\eta_{f}: A_{X}(K) / c(X) \rightarrow A\left(X \cap Z_{K}\right), \quad \eta_{*}: A_{X}(K) / I(K) \rightarrow A\left(X \cap Z_{K}\right) .
$$

The formula

$$
\left(\phi_{\| f} a\right)(\phi k)=\phi[a(k)] \quad\left[\text { for all } a \in A_{X}(K) \text { and all } k \in K\right]
$$

defines a single-valued homomorphism

$$
\phi_{\mathbb{f}}: A_{\mathbf{X}}(K) \rightarrow A(N)
$$

which satisfies

$$
\phi_{H} c_{K}=c_{N} \phi ; \quad \phi_{\#}[c(X)]=1 ; \quad \phi_{\#}[I(K)]=I(N)
$$

and which therefore induces homomorphisms

$$
\phi_{*}: A_{X}(K) / c(X) \rightarrow A(N), \quad \phi_{0}: A_{X}(K) / I(K) \rightarrow A(N) / I(N) .
$$

The homomorphism $c_{K}: K \rightarrow I(K)$ induces a homomorphism of $K / X$ onto $I(K) / c(X)$, which, by virtue of $\phi: K \rightarrow N$, may be regarded as a homomorphism of $N$ onto $I(K) / c(X)$. As such, we denote it by

$$
\psi: N \rightarrow I(K) / c(X) \text {; }
$$

it is characterized by the condition

$$
\psi \phi=\lambda c_{K} .
$$


The homomorphism $C_{N}: G \rightarrow A(N)$ maps $N$ onto $I(N)$, and hence induces a homomorphism

$$
\left(C_{N}\right)_{\ddagger}: Q \rightarrow A(N) / I(N) .
$$

It is easy to verify commutativity relations everywhere in the diagram

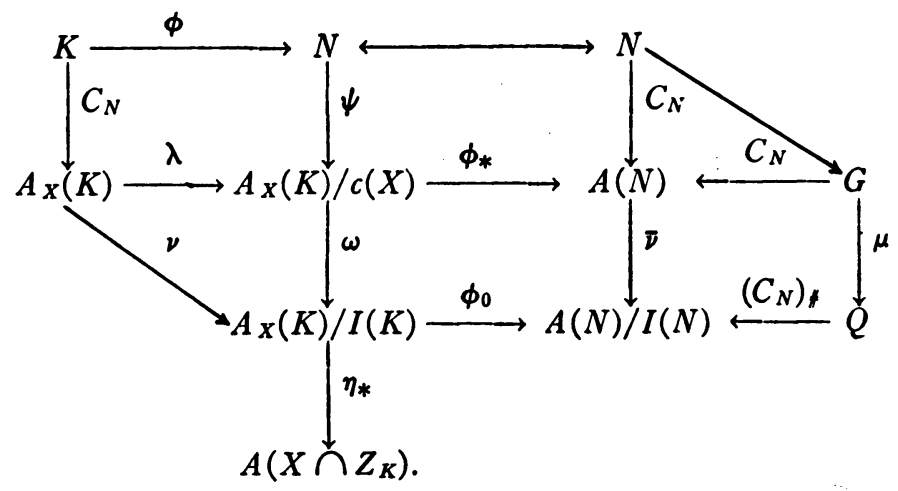

The only maps not explicitly included in (2.6) are the compositions

$$
\eta_{f}=\eta_{*} \omega ; \quad \eta=\eta_{f} \lambda=\eta_{*} \nu ; \quad \phi_{t}=\phi_{*} \lambda .
$$

It can be verified that the horizontal sequences in the commutative diagram

(2.8)

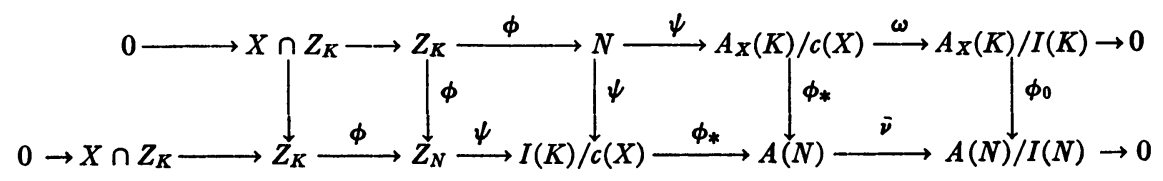

are exact. In particular,

$$
\begin{aligned}
\psi^{-1}(0) & =\phi\left(Z_{K}\right), \\
\psi(N) & =\omega^{-1}(0)=I(K) / c(X), \\
I(K) / c(X) \cap \phi_{*}^{-1}(1) & =\psi\left(Z_{N}\right)=\lambda c_{K}\left[\phi^{-1}\left(Z_{N}\right)\right]=c_{K}\left[\phi^{-1}\left(Z_{N}\right)\right] / c(X) \\
\phi_{*}[I(K) / c(X)] & =\bar{\nu}^{-1}(0)=I(N) .
\end{aligned}
$$

Formula (2.11) is established with the help of

$$
I(K) \cap \phi_{f}^{-1}(1)=c_{K}\left[\phi^{-1}\left(Z_{N}\right)\right] .
$$

It can be shown that for every $a \in A_{X}(K)$ and every $n \in N$,

$$
\psi\left[\left(\phi_{f} a\right) n\right]=\lambda(a)+\psi(n)-\lambda(a) .
$$

It follows that for every $w \in A_{X}(K) / c(X)$ and every $n \in N$,

$$
\psi\left[\left(\phi_{*} w\right) n\right]=w+\psi(n)-w .
$$

Formulae (2.15) and 


$$
\phi_{*} \psi=c_{N},
$$

which is included in the commutativity of (2.6), show that the homomorphisms $\psi: N \rightarrow A_{X}(K) / c(X), \phi_{*}: A_{X}(K) / c(X) \rightarrow A(N)$ constitute a crossed module.

3. Pseudo-modules. Let $(E, \bar{\phi})$ be a continuation of $\phi: K \rightarrow G$. The homomorphism $C_{K}: E \rightarrow A_{X}(K)$ induces a homomorphism of $E / X$ into $A_{X}(K) / c(X)$, which, by virtue of the isomorphism $E / X \approx G$ induced by $\bar{\phi}: E \rightarrow G$, may be regarded as a homomorphism of $G$ into $A_{X}(K) / c(X)$. As such, we denote it $\theta: G \rightarrow A_{X}(K) / c(X)$; it is characterized by the condition

$$
\theta \bar{\phi}=\lambda C_{K} .
$$

We observe

$$
\phi \psi C_{K}=C_{N} \bar{\phi}, \quad \phi_{*} \lambda C_{K}=C_{N} \bar{\phi}
$$

[(3.2a) is an obvious generalization of $(2.4 \mathrm{a})$; (3.2b) follows from (3.2a) and $(2.7 \mathrm{c})]$.

Consider now the diagram

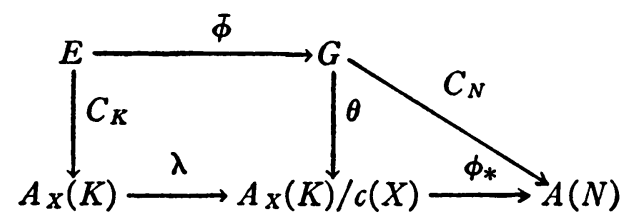

Commutativity holds around the outer perimeter by (3.2b), and in the rectangle by (3.1). Since $\phi(E)=G$, it follows that commutativity holds also in the triangle.

In (3.3), if we restrict $\Phi$ and $C_{K}$ to $K$, we obtain commutativity in the diagram

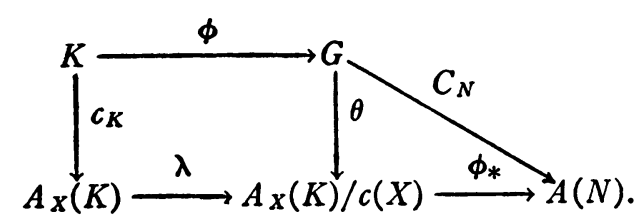

By (2.5), commutativity in the rectangle of (3.4) is equivalent to

$$
\theta \mid N=\psi \text {. }
$$

Definition. A modular structure on the normal homorphism $\phi: K \rightarrow G$ is a homomorphism $\theta: G \rightarrow A_{X}(K) / c(X)$ such that (3.4) is commutative. A pseudo-module is a normal homomorphism together with a modular structure; a pseudo-module will generally be denoted $(\phi, \theta)$.

Remark. Commutativity in the triangle of (3.4) no longer follows auto- 
matically from commutativity in the rectangle and around the perimeter, because, in general, $\phi(K) \neq G$. Hence a modular structure is required to satisfy two independent commutativity relations. These relations represent the least we can demand of $\theta$ in order that it might possibly have arisen from a continuation in the above manner. Moreover, they are exactly the relations needed to insure commutativity throughout (2.6) when $\theta: G \rightarrow A_{X}(K) / c(X)$ is adjoined to the diagram.

An extension of a pseudo-module $(\phi, \theta)$ is a continuation $(E, \bar{\phi})$ of $\phi: K \rightarrow G$ satisfying (3.1). Here, (3.1) is regarded not as the definition of $\theta$ but as a restriction imposed on the desired $\Phi$ in terms of the given $\theta$.

It follows from the above discussion that for every continuation $(E, \bar{\phi})$ of $\phi: K \rightarrow G$, there exists one and only one modular structure $\theta$ on $\phi$ such that $(E, \bar{\phi})$ is an extension of the pseudo-module $(\phi, \theta)$. Thus the continuations divide into classes according to the pseudo-modules of which they are extensions. In particular, a necessary condition for the continuability of a normal homomorphism is that it admit a modular structure.

ExAmpLE. Let $K$ be the nonabelian group of order 6, let $G$ be the cyclic group of order 4 generated by the symbol $g_{0}$, and let $\phi: K \rightarrow G$ be the nontrivial homomorphism. That is, if $k \neq 0$, then $\phi(k)=2 g_{0}$ or 0 according as $k$ is of order 2 or 3. In this case $c_{K}$ is an isomorphism of $K$ onto $I(K)=A_{X}(K)$ $=A(K)$; hence $A_{X}(K) / c(X)$ is of order 2. Suppose $\theta: G \rightarrow A_{X}(K) / c(X)$ is a modular structure. Since $A_{X}(K) / c(X)$ is of order 2, we have $\theta\left(2 g_{0}\right)=2 \theta\left(g_{0}\right)=0$. But choose a nonzero element $k$ of $K$ of order 2. Since $k \notin X$, we have $c(k)$ $\in c(X)$, and $\lambda c(k) \neq 0$. But $\phi(k)=2 g_{0}$. Therefore by commutativity in the rectangle of (3.4), $\theta\left(2 g_{0}\right)=\theta \phi(k)=\lambda c(k) \neq 0$. The contradiction shows that this normal homomorphism admits no modular structure and hence is not continuable; in fact it admits no homomorphism $\theta: G \rightarrow A_{X}(K) / c(X)$ satisfying (3.5).

This paper is mainly devoted to a classification of the extensions of a given pseudo-module. Some information on the complementary problem-the classification of the possible modular structures on a given normal homomorphism-will be given in $\S 11$; more will be found in [10].

A crossed module has been defined by J. H. C. Whitehead [14] as a system consisting of two groups $K$ and $G$, together with two homomorphisms $\phi: K$ $\rightarrow G$ and $\theta: G \rightarrow A(K)$, such that

$$
\begin{gathered}
\theta \phi=c_{K}, \\
\phi[(\theta g) k]=g+\phi(k)-g \quad(\text { for all } g \in G \text { and all } k \in K) .
\end{gathered}
$$

It follows from (3.7) that $\phi: K \rightarrow G$ is a normal homomorphism. If we grant the normality of $N=\phi(K)$, so that $C_{N}: G \rightarrow A(N)$ is well defined, (3.7) may be written

$$
\phi[(\theta g) k]=\left(C_{N} g\right)(\phi k) .
$$


It also follows from (3.7) that $\theta(G) \subset A_{X}(K)$, where, as usual, $X=\phi^{-1}(0)$. If we grant this condition, in addition to the normality of $N$, then by (2.3), formula (3.8) is equivalent to

$$
\phi_{i} \theta=C_{N} .
$$

Hence a crossed module may be defined as a normal homomorphism $\phi: K \rightarrow G$ together with a homomorphism $\theta: G \rightarrow A_{X}(K)$ satisfying (3.6) and (3.9). From (3.6) we obtain $X \subset Z_{K}$. Now, in the definition of a pseudomodule, if we assume $X \subset Z_{K}$, we have $c(X)=1$, and $\lambda$ becomes the identity mapping of $A_{X}(K)$ onto itself. Hence $\theta: G \rightarrow A_{X}(K) / c(X)$ becomes $\theta: G$ $\rightarrow A_{X}(K)$, and (3.4) collapses to conditions (3.6) and (3.9). Therefore a crossed module is the same thing as a pseudo-module in which $X \subset Z_{K}$.

As a special case of the concept of an extension of a pseudo-module, we obtain a definition of an extension [13] of a crossed module $(\phi, \theta)$, as a continuation $(E, \Phi)$ of $\phi: K \rightarrow G$ satisfying

$$
\theta \bar{\phi}=C_{K} .
$$

In the special case $\phi=0$ (not necessarily assuming $X \subset Z_{K}$ ), we have $A_{X}(K) / c(X)=A(K) / I(K)$; both commutativity relations in (3.4) reduce to the requirement that $0=0$. Hence a modular structure on a normal homomorphism of this type is simply an arbitrary homomorphism of $Q(=G)$ into $A(K) / I(K)$. It follows that a $Q$-kernel [5] is the same thing as a pseudomodule in which $\phi=0$. Since we now have $\lambda=\nu$, an extension of such a pseudomodule may be regarded as a group $E$, containing $K$ as a subgroup, together with a homomorphism $\rho(=\mu \Phi)$ of $E$ onto $Q$, with kernel $K$, satisfying

$$
\theta \rho=\nu C_{K} .
$$

Such an extension is the same thing as an extension of the $Q$-kernel $\theta: Q$ $\rightarrow A(K) / I(K)$ in the sense of [5].

The intersection of our two special cases is that in which $\phi=0$ and $K$ is abelian. This leads to the theory of extensions of an arbitrary group $Q$ by an abelian group $K$ [4]; a modular structure is now an arbitrary homomorphism $\theta: Q \rightarrow A(K)$, i.e., an operation of $Q$ on $K$ as a group of operators.

We remark that every extension $(E, \Phi)$ of a pseudo-module $(\phi, \theta)$ can itself be made into a pseudo-module in one and only one way. That is, there exists one and only one homomorphism $\bar{\theta}: G \rightarrow A_{X}(E) / c_{E}(X)$ giving commutativity in

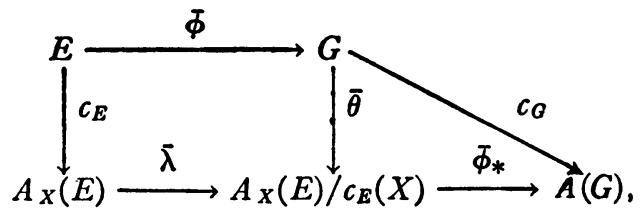


where $\bar{\lambda}: A_{X}(E) \rightarrow A_{X}(E) / c_{E}(X)$ is the natural homomorphism, and $\bar{\phi}_{*}$ : $A_{X}(E) / c_{E}(X) \rightarrow A(G)$ is defined analogously with $\phi_{*}$ (\$2). In fact, in exact analogy with the definition of $\psi: N \rightarrow I(K) / c_{K}(X)$ in $\$ 2$, there exists one and only one homomorphism $\bar{\theta}: G \rightarrow A_{X}(E) / c_{E}(X)$ giving commutativity in the rectangle of (3.12); commutativity in the triangle follows automatically.

Moreover, let $A_{X, K}(E)$ denote the group of all automorphisms of $E$ which map $X$ onto itself and $K$ onto itself; let $\zeta: A_{X, K}(E) \rightarrow A_{X}(K)$ denote the natural homomorphism defined by restricting each element of $A_{X, K}(E)$ to $K$; and let $\zeta_{\sharp}: A_{X, K}(E) / c_{E}(X) \rightarrow A_{X}(K) / c_{K}(X)$ denote the homomorphism induced by $\zeta$. Then $\bar{\theta}$ is an "extension" of $\theta$ in the sense that $\bar{\theta}(G)$ $C A_{X, K}(E) / c_{E}(X)$ and

$$
\zeta \bar{\theta}=\theta .
$$

However, the pseudo-module which arises in this way from an extension of a crossed module is not necessarily a crossed module, because the central subgroup $X$ of $K$ is not necessarily a central subgroup of $E$.

4. Cochains. Let $P$ and $M$ be arbitrary groups. By an $n$-cochain on $P$ in $M$ we mean a function $f$ defined on $P^{n}$ with values in $M$, such that $f\left(p_{1}, \cdots, p_{n}\right)=0$ for every $n$-tuple $\left(p_{1}, \cdots, p_{n}\right)$ which has at least one coordinate zero( $\left.{ }^{5}\right)$. By addition of values in $M$, the set of all $n$-cochains on $P$ in $M$ forms an additive group $C^{n}(P, M)$.

If $u$ is a 1-cochain on $P$ in $M$, its deviation [4] will be denoted dev $u$; it is a 2-cochain on $P$ in $M$, and is defined by

$$
(\operatorname{dev} u)\left(p, p^{\prime}\right)=u(p)+u\left(p^{\prime}\right)-u\left(p+p^{\prime}\right) .
$$

If $P, M, L$ are arbitrary groups, $u$ is a 1-cochain on $P$ in $M$, and $\beta: M \rightarrow L$ is a homomorphism, then

$$
\operatorname{dev}(\beta u)=\beta \operatorname{dev} u .
$$

In particular, suppose $u$ is a 1 -cochain on $P$ in $M$, and $R$ is a normal subgroup of $M$. Let $\beta: M \rightarrow M / R$ denote the natural homomorphism. Then, by (4.2), $\operatorname{dev}(\beta u)=0$ if and only if $\beta \operatorname{dev} u=0$. Hence, $\beta u$ is a homomorphism if and only if $\left({ }^{6}\right) \operatorname{dev} u \subset R$.

In this paper no mappings of one group into another will be considered which are not 1 -cochains. That is, every mapping must carry 0 to 0 ; but a mapping need not be a homomorphism unless specified. In particular, by a right inverse of a homomorphism $\beta: P \rightarrow M$ we mean a 1-cochain $u$ on $M$ in $P$ such that the composition $\beta u$ is the identity mapping of $M$ on itself. Thus a homomorphism admits a "right inverse" if and only if it is onto.

Let $f$ be a 2-cochain on $P$ in $M, t$ a 1-cochain on $P$ in $A(M)$, and $h$ a 1-co-

$\left(^{5}\right)$ Our use of the term $n$-cochain differs slightly from that in [4], in that our cochains are always normalized, and may have values in any group, not necessarily abelian.

(6) Given a mapping $f$ of $A$ into $B$ and a subset $C$ of $B, f \subset C$ means $f(A) \subset C$. 
chain on $P$ in $M$. We define a 2-cochain $h_{t} \cdot f$ on $P$ in $M$ by the formula( $\left.{ }^{7}\right)$

$$
\left(h_{t} \cdot f\right)\left(p, p^{\prime}\right)=h(p)+(t p)\left(h p^{\prime}\right)+f\left(p, p^{\prime}\right)-h\left(p+p^{\prime}\right) .
$$

LemmA (4.4). Let $P$ and $M$ be arbitrary groups; let $L$ and $Y$ be normal subgroups of $M$, with $Y \subset Z_{L}$; let $\eta: A_{Y}(L) \rightarrow A(Y), C_{L}: M \rightarrow A_{Y}(L), C_{Y}: M \rightarrow A(Y)$ be the natural homomorphisms. Let $f$ be a 2-cochain on $P$ in $L, h$ a 1-cochain on $P$ in $L, u$ a 1-cochain on $P$ in $M$ such that dev $u \subset L$, and $t$ a 1-cochain on $P$ in $A_{Y}(L)$ such that dev $t \subset I(L)$. Then

$$
h_{C_{L} u} \cdot \operatorname{dev} u=\operatorname{dev}(h+u) \text {. }
$$

If $h \subset Y$ and $\delta: C^{1}(P, Y) \rightarrow C^{2}(P, Y)$ is the coboundary homomorphism [4] associated with the homomorphism $\eta t: P \rightarrow A(Y)$, then

$$
h_{t} \cdot f=\delta h+f \text {. }
$$

If $h \subset Y$, and $\delta: C^{1}(P, Y) \rightarrow C^{2}(P, Y)$ is the coboundary homomorphism associated with the homomorphism $C_{Y} u: P \rightarrow A(Y)$, then

$$
\operatorname{dev}(h+u)=\delta h+\operatorname{dev} u \text {. }
$$

Proof. (4.5) is easily verified by direct calculation. (4.6), under the hypotheses $h \subset Y \subset Z_{L}$, follows from a comparison of (4.3) with the (nonhomogeneous) coboundary formula of [4].

Since $C_{Y}=\eta C_{L}$, the coboundary homomorphism considered in (4.7) is associated with $\eta C_{L} u: P \rightarrow A(Y)$. But dev $\left(C_{L} u\right)=C_{L} \operatorname{dev} u=c_{L} \operatorname{dev} u \subset I(L)$. Therefore by (4.6), with $C_{L} u$ in the role of $t$ and dev $u$ in the role of $f$, we have $h_{C_{L^{u}}} \cdot \operatorname{dev} u=\delta h+\operatorname{dev} u$. Combining this with (4.5), we obtain (4.7). Q.E.D.

Let $f$ and $g$ be 2 -cochains on $P$ in $M$, and let $t$ be a 1 -cochain on $P$ in $A(M)$. If $g \subset Z_{M}$, then clearly

$$
h_{t} \cdot(g+f)=g+h_{t} \cdot f .
$$

It follows that if $f$ and $f^{\prime}$ are 2-cochains on $P$ in $M$, such that $f^{\prime}-f \subset Z_{M}$, then

$$
h_{t} \cdot f^{\prime}-h_{t} \cdot f=f^{\prime}-f .
$$

Given a 2-cochain $f$ on $P$ in $M$ and a 1-cochain $t$ on $P$ in $A(M)$, we define a 3 -cochain $\delta_{t} f$ on $P$ in $M$ by the formula $\left({ }^{8}\right)$

$$
\begin{aligned}
\left(\delta_{t} f\right)\left(p, p^{\prime}, p^{\prime \prime}\right)= & (t p) f\left(p^{\prime}, p^{\prime \prime}\right)+f\left(p, p^{\prime}+p^{\prime \prime}\right) \\
& -f\left(p+p^{\prime}, p^{\prime \prime}\right)-f\left(p, p^{\prime}\right) .
\end{aligned}
$$

For fixed $t$, the mapping $\delta_{t}$ is a 1 -cochain on $C^{2}(P, M)$ in $C^{3}(P, M)$. In

(7) Cf. [5, formula (7.3)]; also [8].

(8) Cf. [5, formula (7.2)]. Also, compare our formula (5.4a) with formula (7.1) of [5]. See, in fact, [8]. 
general, $\delta_{t}$ is not a homomorphism. However, if $f$ and $f^{\prime}$ are 2-cochains on $P$ in $M$, and $f \subset Z_{M}$, then, just as in the abelian case,

$$
\delta_{t}\left(f+f^{\prime}\right)=\delta_{t} f+\delta_{t} f^{\prime} .
$$

It follows that if $f$ and $f^{\prime}$ are 2-cochains on $P$ in $M$ such that $f^{\prime}-f \subset Z_{M}$, then

$$
\delta_{t}\left(f^{\prime}-f\right)=\delta_{t} f^{\prime}-\delta_{t} f .
$$

Let $Y$ be a subgroup of $Z_{M}$; let $t$ be a 1-cochain on $P$ in $A_{Y}(M)$, such that dev $t \subset I(M)$; let $\eta: A_{Y}(M) \rightarrow A(Y)$ denote the natural homomorphism, and let $\delta: C^{2}(P, Y) \rightarrow C^{3}(P, Y)$ denote the coboundary homomorphism [4] associated with the homomorphism $\eta t: P \rightarrow A(Y)$. If $f$ is a 2-cochain on $P$ in $M$ such that $f \subset Y$, then clearly $\delta_{t} f=\delta f$. Hence (4.11) and (4.12) can be stated as follows:

Lemma (4.13). Let $P$ and $M$ be arbitrary groups; let $Y, t, \eta, \delta$ be as in the preceding paragraph; let $f$ and $f^{\prime}$ be 2-cochains on $P$ in $M$. If $f \subset Y$, then $\delta_{t}\left(f+f^{\prime}\right)=\delta f+\delta_{t} f^{\prime}$. On the other hand, if $f^{\prime}-f \subset Y$, then $\delta\left(f^{\prime}-f\right)=\delta_{t} f^{\prime}-\delta_{t} f$.

Further elementary facts on cochains with nonabelian coefficients will be incorporated in $\$ 5$. For abelian coefficients, we shall follow [4], using normalized nonhomogeneous cochains throughout. The symbol $\delta f$ will be used only when the values of $f$ lie in an abelian group, on which $P$ operates in a selfevident fashion.

5. The case $\phi=0$. In this section we review, in a form convenient for later reference in this and subsequent papers, the results of [5] on group extensions by a non-abelian kernel; see also [2, 8]. We shall omit proofs, since they can be extracted from [5]; see remark following Corollary 5.18.

Let $Q$ and $K$ be arbitrary groups. An extension of $Q$ by $K$ is a group $E$, containing $K$ as a subgroup, together with a homomorphism $\rho: E \rightarrow Q$ such that the sequence

$$
0 \rightarrow K \rightarrow E \stackrel{\rho}{\rightarrow} Q \rightarrow 0
$$

is exact.

Lemma (5.2). Let $(E, \rho)$ be an extension of $Q$ by $K$. Let $d$ be a right inverse of $\rho: E \rightarrow Q$. Define

$$
f=\operatorname{dev} d, \quad t=C_{K} d .
$$

Then $f \subset K$, and

$$
c_{K} f=\operatorname{dev} t, \quad \delta_{t} f=0 .
$$

Definition. A structural cocycle on $Q$ in $K$ is an ordered pair $(f, t)$, where $f$ is a 2-cochain on $Q$ in $K$, and $t$ is a 1-cochain on $Q$ in $A(K)$, satisfying (5.4). 
Let $(f, t)$ be a structural cocycle on $Q$ in $K$, and let $(E, \rho)$ be an extension of $Q$ by $K$. We say that $(f, t)$ represents $(E, \rho)$ if there exists a right inverse $d$ of $\rho: E \rightarrow Q$ satisfying (5.3). By Lemma 5.2, every extension is represented by at least one structural cocycle. Conversely,

Lemma (5.5). Every structural cocycle on $Q$ in $K$ represents at least one extension of $Q$ by $K$.

Lemma (5.6). If $(f, t)$ and $\left(f^{\prime}, t^{\prime}\right)$ are structural cocycles representing the same extension $(E, \rho)$, then there exists a 1-cochain $h$ on $Q$ in $K$ such that

$$
f^{\prime}=h_{t} \cdot f, \quad t^{\prime}=c h+t .
$$

Specifically, if $d^{\prime}$ and $d$ are right inverses of $\rho: E \rightarrow Q$ satisfying (5.3) with and without primes, respectively, then $h=d^{\prime}-d$ satisfies (5.7).

Two structural cocycles $(f, t)$ and $\left(f^{\prime}, t^{\prime}\right)$ will be called equivalent if there exists a 1-cochain $h$ on $Q$ in $K$ satisfying (5.7). This relation is reflexive, symmetric, and transitive. The set of all equivalence classes of structural cocycles on $Q$ in $K$ will be denoted $\bar{H}^{2}(Q, K)$; no "addition" is defined in this set.

THEOREM (5.8). The relation " $(f, t)$ represents $(E, \rho)$ " induces a 1-1 correspondence between $\bar{H}^{2}(Q, K)$ and the set of all isomorphism-classes of extensions of $Q$ by $K$.

In Theorem 5.8, two extensions $(E, \rho)$ and $\left(E^{\prime}, \rho^{\prime}\right)$ are considered isomorphic if there exists an isomorphism $W: E^{\prime} \approx E$ which maps $K$ identically onto itself and satisfies $\rho W=\rho^{\prime}$.

If $(E, \rho)$ is an extension of $Q$ by $K$, then there exists one and only one homomorphism $\theta: Q \rightarrow A(K) / I(K)$ satisfying (3.11). Any homomorphism of the form $\theta: Q \rightarrow A(K) / I(K)$ is called a $Q$-kernel. By an extension of a $Q$-kernel $\theta: Q \rightarrow A(K) / I(K)$ we mean an extension of $Q$ by $K$ satisfying (3.11). Henceforth we restrict attention mainly to a fixed $Q$-kernel $\theta: Q \rightarrow A(K) / I(K)$.

The natural homomorphism $\eta: A(K) \rightarrow A\left(Z_{K}\right)$ carries $I(K)$ to the identity element of $A\left(Z_{K}\right)$ and hence induces a homomorphism $\eta_{*}: A(K) / I(K)$ $\rightarrow A\left(Z_{K}\right)$. The cohomology groups $H^{n}\left(Q, Z_{K}\right)$ are defined relative to the operation $\eta_{*} \theta: Q \rightarrow A\left(Z_{K}\right)$ of $Q$ on $Z_{K}$.

By a structural cocycle in $\theta: Q \rightarrow A(K) / I(K)$ we mean a structural cocycle $(f, t)$ on $Q$ in $K$ such that $\nu t=\theta$. Every structural cocycle on $Q$ in $K$ is a structural cocycle in one and only one $Q$-kernel; equivalent structural cocycles on $Q$ in $K$ lie in the same $Q$-kernel. The set of all equivalence classes of structural cocycles in $\theta: Q \rightarrow A(K) / I(K)$ is denoted $\bar{H}^{2}(Q, K, \theta)$; this set may be empty.

Lemma (5.9). Let $(f, t)$ be a structural cocycle on $Q$ in $K$ representing an extension $(E, \rho)$ of $Q$ by $K$. Then the $Q-k e r n e l$ of which $(E, \rho)$ is an extension is the same as the Q-kernel to which $(f, t)$ belongs. 
Combining Lemma 5.9 with Theorem 5.8, we have

TheOREM (5.10). The relation " $(f, t)$ represents $(E, \rho)$ " induces a 1-1 correspondence between $\bar{H}^{2}(Q, K, \theta)$ and the set of all isomorphism-classes of extensions of $\theta: Q \rightarrow A(K) / I(K)$.

Given any structural cocycle $(f, t)$ in $\theta: Q \rightarrow A(K) / I(K)$, and any $g$ $\in Z^{2}\left(Q, Z_{K}\right)$, we define

$$
g+(f, t)=(g+f, t) .
$$

Then $g+(f, t)$ is a structural cocycle in $\theta: Q \rightarrow A(K) / I(K)$.

Theorem (5.12). Formula (5.11) induces an operation of $H^{2}\left(Q, Z_{K}\right)$ as a simply transitive transformation group on $\bar{H}^{2}(Q, K, \theta)$, and hence, via Theorem 5.10 , on the set of all isomorphism-classes of extensions of $\theta: Q \rightarrow A(K) / I(K)$.

Corollary (5.13). If $\theta: Q \rightarrow A(K) / I(K)$ is extendible, there exists a 1-1 correspondence (not "natural," in general) between the cohomology group $H^{2}\left(Q, Z_{K}\right)$ and the set of all isomorphism-classes of extensions of $\theta: Q$ $\rightarrow A(K) / I(K)$.

Definition. A structural cochain in $\theta: Q \rightarrow A(K) / I(K)$ is an ordered pair $(f, t)$, where $f$ is a 2-cochain on $Q$ in $K$, and $t$ is a 1-cochain on $Q$ in $A(K)$ satisfying $c f=\operatorname{dev} t$ and $\nu t=\theta$, but not necessarily $\delta_{t} f=0$. A $Q$-kernel need not admit a structural cocycle, but it always admits a structural cochain.

We define the coboundary $\delta(f, t)$ of a structural cochain $(f, t)$ by

$$
\delta(f, t)=\delta_{t} f \in C^{3}(Q, K) .
$$

We say that two structural cochains $(f, t)$ and $\left(f^{\prime}, t^{\prime}\right)$ are equivalent if there exists a 1 -cochain $h$ on $Q$ in $K$ satisfying (5.7).

Lemma (5.15). If $(f, t)$ and $\left(f^{\prime}, t^{\prime}\right)$ are structural cochains in $\theta: Q$ $\rightarrow A(K) / I(K)$, then there exists a 2-cochain $f^{\prime \prime}$ on $Q$ in $K$ such that $\left(f^{\prime \prime}, t\right)$ is a structural cochain equivalent to $\left(f^{\prime}, t^{\prime}\right)$.

Lemma (5.16). Any two equivalent structural cochains have the same coboundary.

Lemma 5.16 may be thought of as the generalization to non-abelian coefficients of the proposition that the double coboundary of a 1-cochain is zero.

THEOREM (5.17). Let $\bar{C}^{2}(Q, K, \theta)$ denote the set of all structural cochains in $\theta: Q \rightarrow A(K) / I(K)$. The mapping $\delta: \bar{C}^{2}(Q, K, \theta) \rightarrow C^{3}(Q, K)$ defined by $(5.14)$ maps $\bar{C}^{2}(Q, K, \theta)$ into, and onto, a single coset $\left(^{9}\right)$ of $B^{3}\left(Q, Z_{K}\right)$ in $Z^{3}\left(Q, Z_{K}\right)$.

The cohomology class $\delta\left[\bar{C}^{2}(Q, K, \theta)\right]$, regarded as an element of $H^{3}\left(Q, Z_{K}\right)$,

(9) If $A$ is a normal subgroup of $B$, we speak of the "cosets of $A$ in $B$," meaning the classes which form the elements of $B / A$; some would call these the "cosets of $B \bmod A . "$ 
is called the obstruction $\left({ }^{10}\right)$ of the $Q$-kernel $\theta: Q \rightarrow A(K) / I(K)$.

By Lemmas 5.2, 5.5, and 5.9, $\theta: Q \rightarrow A(K) / I(K)$ is extendible if and only if it admits a structural cocycle. But by Theorem 5.17 and the definition of the obstruction, $\theta: Q \rightarrow A(K) / I(K)$ admits a structural cocycle if and only if its obstruction vanishes. Hence

Corollary (5.18). A Q-kernel is extendible if and only if its obstruction vanishes.

REMARK ON PROOFS. The proof of Lemma 5.5 consists mainly of defining addition in the set $K \times Q$ by the formula $(k, q)+\left(k^{\prime}, q^{\prime}\right)=\left[k+(t q) k^{\prime}+f\left(q, q^{\prime}\right)\right.$, $\left.q+q^{\prime}\right]$ and showing that conditions (5.4) imply the associative law; this is included in the proof of Theorem 8.1 of [5], and is also indicated in [8]. Lemma 5.16 is not explicitly stated in [5]; in fact the notion of "equivalence" is not brought into the open. However, our formula (4.3) is given as formula (7.3) of [5], and the calculation which proves Lemma 5.16 is included in the proof of Lemma 7.3 in [5]. Our lemmas 4.13, 5.15, and 5.16 are all used in the proof of Theorem 5.17.

We append here some technical lemmas on homomorphisms of one group extension into another.

Lemma (5.19). Let $(E, \rho)$ be an extension of $Q$ by $K$. Let $E^{\prime}$ be a group, and $\phi: K \rightarrow E^{\prime}$ a homomorphism. Let $d$ be a right inverse of $\rho: E \rightarrow Q$, and $v$ a 1-cochain on $Q$ in $E^{\prime}$. There exists at most one homomorphism $\phi: E \rightarrow E^{\prime}$ satisfying

$$
\bar{\phi} \mid K=\phi, \quad \bar{\phi} d=v .
$$

Moreover, there exists such a homomorphism if and only if $\phi(K)$ is a normal subgroup of the group spanned by itself and $v(Q)$, and

$$
\phi \operatorname{dev} d=\operatorname{dev} v, \quad \phi_{f} C_{K} d=C_{K^{\prime}} v,
$$

where $K^{\prime}=\phi(K)$, and $\phi_{f}: A_{\phi^{-1}(0)}(K) \rightarrow A\left(K^{\prime}\right)$ is the homomorphism induced by $\phi: K \rightarrow K^{\prime}$ in the manner of $\$ 2$.

Proof. Suppose conditions (5.21) [and by implication, the assertion about the normality of $\phi(K)]$ are satisfied. Every element of $E$ is uniquely expressible in the form $k+d(q)$, where $k \in K$ and $q \in Q$. We define $\Phi[k+d(q)]$ $=\phi(k)+v(q)$. Then

$$
\begin{aligned}
\Phi\left[k+d(q)+k^{\prime}\right. & \left.+d\left(q^{\prime}\right)\right] \\
& =\Phi\left[k+\left(C_{K} d q\right) k^{\prime}+(\operatorname{dev} d)\left(q, q^{\prime}\right)+d\left(q+q^{\prime}\right)\right] \\
& =\phi(k)+\left(\phi_{f} C_{K} d q\right)\left(\phi k^{\prime}\right)+(\phi \operatorname{dev} d)\left(q, q^{\prime}\right)+v\left(q+q^{\prime}\right) \\
& =\phi(k)+\left(C_{K^{\prime}} v q\right)\left(\phi k^{\prime}\right)+(\operatorname{dev} v)\left(q, q^{\prime}\right)+v\left(q+q^{\prime}\right) \\
& =\phi(k)+v(q)+\phi\left(k^{\prime}\right)+v\left(q^{\prime}\right)=\Phi[k+d(q)]+\Phi\left[k^{\prime}+d\left(q^{\prime}\right)\right],
\end{aligned}
$$

${ }^{(10)}$ Sometimes called characteristic cohomology class. 
showing that $\Phi$ is a homomorphism. We leave the remainder of the proof to the reader.

Lemma (5.22). Let $E, \rho, Q, K, E^{\prime}, \phi, d$, v be as in Lemma 5.19, satisfying (5.21). Let $d^{\prime}, v^{\prime}$ satisfy similar properties to those of $d$, v. Let $\Phi: E \rightarrow E^{\prime}$ be the homomorphism satisfying (5.20). Let $\bar{\phi}^{\prime}: E \rightarrow E^{\prime}$ be the homomorphism satisfying $\Phi^{\prime} \mid K=\phi, \Phi^{\prime} d^{\prime}=v^{\prime}$. Then $\Phi=\Phi^{\prime}$ if and only if

$$
v^{\prime}-v=\phi\left(d^{\prime}-d\right) \text {. }
$$

Proof. If $\bar{\phi}=\bar{\phi}^{\prime}$, then $v^{\prime}-v=\bar{\phi} d^{\prime}-\bar{\phi} d=\bar{\phi}\left(d^{\prime}-d\right)=\phi\left(d^{\prime}-d\right)$. If $v^{\prime}-v$ $=\phi\left(d^{\prime}-d\right)$, then $\Phi^{\prime} d^{\prime}-\bar{\phi} d=\phi\left(d^{\prime}-d\right)=\bar{\phi}\left(d^{\prime}-d\right)=\bar{\phi} d^{\prime}-\bar{\phi} d$. Hence

$$
\Phi^{\prime} d^{\prime}=\Phi d^{\prime}, \text { and } \Phi\left|d^{\prime}(Q)=\Phi^{\prime}\right| d^{\prime}(Q) \text {. }
$$

Since also $\Phi\left|K=\Phi^{\prime}\right| K$, we have $\Phi=\Phi^{\prime}$. Q.E.D.

LemMA (5.24). Let $(f, t)$ be a structural cocycle on $Q$ in $K$, representing an extension $(E, \rho)$ of $Q$ by $K$; let $d$ be a right inverse of $\rho: E \rightarrow Q$ satisfying (5.3). Let $f^{\prime}, t^{\prime}, Q^{\prime}, K^{\prime}, E^{\prime}, \rho^{\prime}, d^{\prime}$ be similar. Let $\phi: K \rightarrow K^{\prime}$ and $\theta: Q \rightarrow Q^{\prime}$ be homomorphisms, with $\phi(K)=K^{\prime}$. Let $r$ be a 1-cochain on $Q$ in $K^{\prime}$. Then there exists at most one homomorphism $\bar{\phi}: E \rightarrow E^{\prime}$ satisfying

$$
\Phi \mid K=\phi, \quad \theta \rho=\rho^{\prime} \Phi, \quad \Phi d=r+d^{\prime} \theta .
$$

Moreover, there exists such a homomorphism if and only if

$$
\phi f=r_{t^{\prime} \theta} \cdot\left(f^{\prime} \theta\right), \quad \phi_{\nexists f} t=c_{K^{\prime}} r+t^{\prime} \theta,
$$

where $\theta\left(q_{1}, q_{2}\right)=\left(\theta q_{1}, \theta q_{2}\right)$ in the right-hand side of (5.26a), and $\phi_{\#}$ is defined as usual. If $\bar{\phi}: E \rightarrow E^{\prime}$ is a homomorphism satisfying (5.25a) and (5.25c), then (5.25b) holds automatically.

This follows easily from Lemma 5.19, on setting $v=r+d^{\prime} \theta$.

6. Reduction of pseudo-modules to crossed modules. Let $L, M, P$ be groups; let $\tau: P \rightarrow L$ and $\beta: M \rightarrow L$ be homomorphisms, with $\beta(M)=L$. Under these circumstances, we define the graph of $\tau$ rel. $\beta$ as the quartet $\left(\Gamma, \epsilon, \pi_{1}, \pi_{2}\right)$, where

(a) $\Gamma$ is the subgroup of the direct sum $P+M$ consisting of those ordered pairs $(p, m)$ which satisfy

$$
\tau(p)=\beta(m) ;
$$

(b) $\epsilon: \beta^{-1}(0) \rightarrow \Gamma, \pi_{1}: \Gamma \rightarrow P, \pi_{2}: \Gamma \rightarrow m$ are the homomorphisms defined by

$$
\epsilon(m)=(0, m) ; \quad \pi_{1}(p, m)=p ; \quad \pi_{2}(p, m)=m .
$$

This definition is based on Baer's concept of the "graph" of a $Q$-kernel( $\left.{ }^{11}\right)$; it will be further developed in [9].

(11) [2]; see also $[5, \S 12]$, and $[6, \S 3]$. 
Now let $(\phi, \theta)$ be an arbitrary pseudo-module; let $\left(\Gamma, \epsilon, \pi_{1}, \pi_{2}\right)$ be the graph of $\theta: G \rightarrow A_{X}(K) / c(X)$ rel. $\lambda: A_{X}(K) \rightarrow A_{X}(K) / c(X)$. By commutativity in the rectangle of (3.4), the homomorphism

$$
\phi^{\prime}: K \rightarrow G+A_{X}(K)
$$

defined by

$$
\phi^{\prime}(k)=\left[\phi(k), c_{K}(k)\right]
$$

maps $K$ into $\Gamma$.

Lemma (6.4). The sequence

$$
0 \longrightarrow X \cap Z_{K} \longrightarrow K \stackrel{\phi^{\prime}}{\rightarrow} \Gamma \stackrel{\mu \pi_{1}}{\rightarrow} Q \longrightarrow 0
$$

is exact.

Proof. $\phi^{\prime-1}(0)=\phi^{-1}(0) \cap c_{K}^{-1}(1)=X \cap Z_{K}$. From $\lambda\left[A_{X}(K)\right]=A_{X}(K) / c(X)$ it follows that $\pi_{1}(\Gamma)=G$, and hence that $\mu \pi_{1}(\Gamma)=Q$.

The assertion ${ }^{\circ} \phi^{\prime}(K)=\left(\mu \pi_{1}\right)^{-1}(0)$ means that given $n \in N$ and $a \in A_{X}(K)$, the relation $\psi(n)=\lambda(a)$ holds if and only if there exists $k \in K$ such that $\phi(k)=n$ and $c_{K}(k)=a$. If $a \notin I(K)$, both propositions whose equivalence is asserted are false. For $a \in I(K)$, the desired result follows easily from a consideration of the following commutative diagram, in which the horizontal sequences are exact and the vertical homomorphisms are onto:

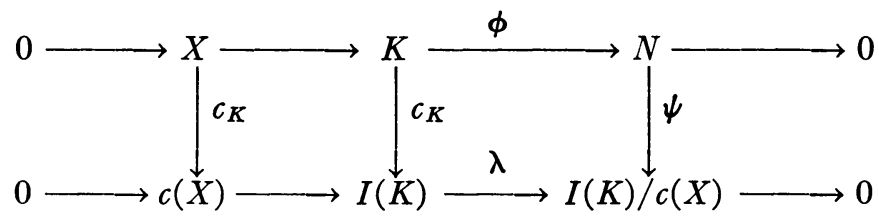

Q.E.D.

THEOREM (6.7). The homomorphisms

$$
\phi^{\prime}: K \rightarrow \Gamma, \pi_{2}: \Gamma \rightarrow A_{X}(K)
$$

constitute a crossed module [note that $\left.A_{X}(K) \subset A_{X} \cap z_{K}(K)\right]$. The extensions of $(\phi, \theta)$ are in a natural 1-1 correspondence with the extensions of $\left(\phi^{\prime}, \pi_{2}\right)$.

Proof. Condition (3.6) becomes, in the present notation, $\pi_{2} \phi^{\prime}=c_{K}$, which follows immediately from the definitions.

Consider any $(g, a) \in \Gamma$ and any $k \in K$. Then $\phi(a k)=\left(\phi_{*} a\right)(\phi k)=\left(\phi_{*} \lambda a\right)(\phi k)$ $=\left(\phi_{*} \theta g\right)(\phi k)=\left(C_{N} g\right)(\phi k)$, and $c_{K}(a k)=a\left(c_{K} k\right) a^{-1}$. Therefore $\phi^{\prime}\left[\pi_{2}(g, a) \cdot k\right]$ $=\phi^{\prime}(a k)=\left[\phi(a k), c_{K}(a k)\right]=\left[\left(C_{N} g\right)(\phi k), a\left(c_{K} k\right) a^{-1}\right]=(g, a)+\phi^{\prime}(k)-(g, a)$, establishing the appropriate version of $(3.7)$, and proving that $\left(\phi^{\prime}, \pi_{2}\right)$ is a crossed module.

Let $\left(E, \bar{\phi}^{\prime}\right)$ be an extension of $\left(\phi^{\prime}, \pi_{2}\right)$; let $N^{\prime}$ denote $\left(\mu \pi_{1}\right)_{1}^{-1}(0)=\pi_{1}^{-1}(N) \subset \Gamma$. Then, from a consideration of the commutative diagram 


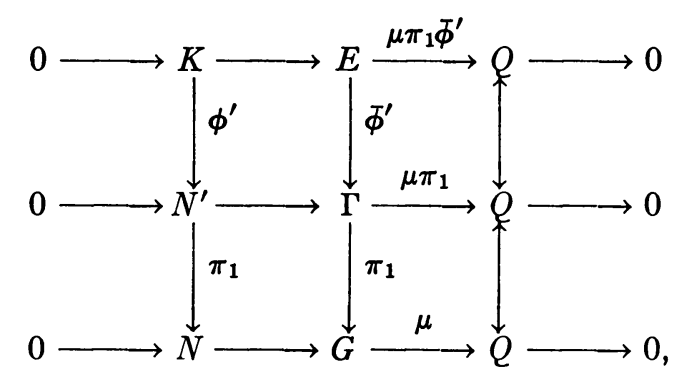

in which the rows are exact, we see that $\left(E, \pi_{1} \bar{\phi}^{\prime}\right)$ is a continuation of $\phi: K \rightarrow G$. Moreover, $\theta \pi_{1} \bar{\phi}^{\prime}=\lambda \pi_{2} \bar{\phi}^{\prime}=\lambda C_{K}$; hence $\left(E, \pi_{1} \bar{\phi}^{\prime}\right)$ is an extension of $(\phi, \theta)$.

Conversely, let $(E, \bar{\phi})$ be an extension of $(\phi, \theta)$. Define $\bar{\phi}^{\prime}: E \rightarrow \Gamma$ by

$$
\bar{\phi}^{\prime}(e)=\left[\bar{\phi}(e), C_{K}(e)\right] .
$$

Condition (3.1) guarantees $\bar{\phi}^{\prime}(e) \in \Gamma$. Comparing (6.9) with (6.3), and using (1.4a), we obtain $\bar{\phi}^{\prime} \mid K=\phi^{\prime}$. Hence commutativity holds throughout (6.8). By (6.9), $\pi_{1} \bar{\phi}^{\prime}=\bar{\phi}$. Therefore $\left(\mu \pi_{1} \bar{\phi}^{\prime}\right)^{-1}(0)=\bar{\phi}^{-1}(N)=K$; hence horizontal exactness holds throughout (6.8). Therefore $\left(E, \phi^{\prime}\right)$ is a continuation of $\phi^{\prime}: K \rightarrow \Gamma$. By $(6.9), \pi_{2} \bar{\phi}^{\prime}=C_{K}$; therefore $\left(E, \bar{\phi}^{\prime}\right)$ is an extension of $\left(\phi^{\prime}, \pi_{2}\right)$.

It is easily verified that these two constructions are inverses of each other. Q.E.D.

Corollary (6.10). The isomorphism-classes of extensions of the pseudomodule $(\phi, \theta)$ are in a natural 1-1 correspondence with the isomorphism-classes of extensions of the crossed module $\left(\phi^{\prime}, \pi_{2}\right)$.

Corollary (6.11). The pseudo-module $(\phi, \theta)$ is extendible if and only if the crossed module $\left(\phi^{\prime}, \pi_{2}\right)$ is extendible.

7. Extensions of crossed modules: $\bar{H}^{2}(\phi, \theta)$. Throughout $\S \S 7-9$, we shall consider a fixed crossed module $(\phi, \theta)$; that is, a pseudo-module in which $X \subset Z_{K}$.

Lemma (7.1). Let $(E, \Phi)$ be an extension of $(\phi, \theta)$, and let $d$ be a right inverse of $\mu \bar{\phi}: E \rightarrow Q$. Define

$$
f=\operatorname{dev} d, \quad u=\Phi d .
$$

Then $f \subset K ; u$ is a right inverse of $\mu: G \rightarrow Q$; and

$$
\phi f=\operatorname{dev} u, \quad \delta_{\theta u} f=0 .
$$

Proof. Since $d$ is a right inverse of $\mu \bar{\phi}, \bar{\phi} d=u$ is a right inverse of $\mu$. Therefore $\operatorname{dev} u \subset \mu^{-1}(0)=N . \phi f=\bar{\phi} \operatorname{dev} d=\operatorname{dev}(\bar{\phi} d)=\operatorname{dev} u \subset N$; therefore, by (1.5b), $f \subset K . \phi f=\Phi f=\operatorname{dev} u$.

Clearly $(E, \mu \overline{)})$ is an extension of $Q$ by $K$. By (3.10), $C_{K} d=\theta \bar{\phi} d=\theta u$. Therefore by (5.4b) of Lemma 5.2, $\delta_{\theta u} f=0$. Q.E.D.

A structural cocycle in $(\phi, \theta)$ is an ordered pair $(f, u)$, where $f$ is a 2-cochain 
on $Q$ in $K$, and $u$ is a right inverse of $\mu: G \rightarrow Q$, satisfying (7.3). Let $(f, u)$ be a structural cocycle in $(\phi, \theta)$, and let $(E, \bar{\phi})$ be an extension of $(\phi, \theta)$. We say that $(f, u)$ represents $(E, \bar{\phi})$ if there exists a right inverse $d$ of $\mu \bar{\phi}: E \rightarrow Q$ satisfying(7.2).

By Lemma 7.1, every extension is represented by at least one structural cocycle. Conversely,

LEMмa (7.4). Every structural cocycle in $(\phi, \theta)$ represents at least one extension of $(\phi, \theta)$.

Proof. Let $(f, u)$ be a structural cocycle in $(\phi, \theta)$. Then $\operatorname{dev}(\theta u)=\theta \operatorname{dev} u$ $=\theta \phi f=c_{K} f$, and $\delta_{\theta u} f=0$. Therefore $(f, \theta u)$ is a structural cocycle on $Q$ in $K$. Hence, by Lemma 5.5, $(f, \theta u)$ represents an extension $(E, \rho)$ of $Q$ by $K$. Choose a right inverse $d$ of $\rho: E \rightarrow Q$ such that $\left(\operatorname{dev} d, C_{K} d\right)=(f, \theta u)$. Then $\phi \operatorname{dev} d=\phi f=\operatorname{dev} u$, and $\phi_{\sharp} C_{K} d=\phi_{\sharp} \theta u=C_{N} u$. Therefore, by Lemma (5.19), there exists one and only one homomorphism $\bar{\phi}: E \rightarrow G$ satisfying (1.4a) and (7.2b). The remaining parts of (1.4) are easily verified. By (7.2b), $\theta \bar{\phi} d=\theta u$ $=C_{K} d$; hence $\theta \bar{\phi}\left|d(Q)=C_{K}\right| d(Q)$. But also $\theta \bar{\phi}\left|K=\theta \phi=c_{K}=C_{K}\right| K$. Therefore $\theta \bar{\phi}=C_{K}$. Q.E.D.

Corollary (7.5). A crossed module is extendible if and only if it admits a structural cocycle.

Lemma (7.6). If $(f, u)$ and $\left(f^{\prime}, u^{\prime}\right)$ are structural cocycles representing the same extension $(E, \Phi)$ of $(\phi, \theta)$, then there exists a 1-cochain $h$ on $Q$ in $K$ such that

$$
f^{\prime}=h_{\theta u} \cdot f, \quad u^{\prime}=\phi h+u .
$$

Specifically, if $d^{\prime}$ and $d$ are right inverses of $\mu \Phi: E \rightarrow Q$ satisfying (7.2) with and without primes, respectively, then $h=d^{\prime}-d$ satisfies (7.7).

Proof. Choose $d^{\prime}, d$, and $h=d^{\prime}-d$ as in the last part of the lemma. Then $\theta u=\theta \bar{\phi} d=C_{K} d$; similarly, $\theta u^{\prime}=C_{K} d^{\prime}$. Therefore $(f, \theta u)$ and $\left(f^{\prime}, \theta u^{\prime}\right)$ are structural cocycles on $Q$ in $K$ representing the extension $(E, \mu \Phi)$ of $Q$ by $K$, via $d$ and $d^{\prime}$ respectively. Therefore, by $(5.7 \mathrm{a}), f^{\prime}=h_{\theta u} \cdot f . u^{\prime}-u=\bar{\phi}\left(d^{\prime}-d\right)=\bar{\phi} h$ $=\phi h$. Q.E.D.

Two structural cocycles $(f, u)$ and $\left(f^{\prime}, u^{\prime}\right)$ in $(\phi, \theta)$ will be called equivalent if there exists a 1-cochain $h$ on $Q$ in $K$ satisfying (7.7). It is easily verified that this relation is reflexive, symmetric, and transitive. The set of all equivalence classes of structural cocycles in $(\phi, \theta)$ will be denoted $\bar{H}^{2}(\phi, \theta)$. Since we never regard the empty set as an equivalence class, the set $\bar{H}^{2}(\bar{\phi}, \theta)$ is non-empty if and only if $(\phi, \theta)$ admits a structural cocycle: i.e. (by Corollary 7.5), if and only if $(\phi, \theta)$ is extendible.

LEMMA (7.8). If $(f, u)$ is a structural cocycle in $(\phi, \theta)$ representing an extension $(E, \bar{\phi})$ of $(\phi, \theta)$, and $\left(E^{\prime}, \bar{\phi}^{\prime}\right)$ is an extension isomorphic to $(E, \phi)$, then 
$(f, u)$ represents $\left(E^{\prime}, \bar{\phi}^{\prime}\right)$.

Proof. Choose a right inverse $d$ of $\mu \bar{\phi}: E \rightarrow Q$ satisfying (7.2), and an isomorphism $W: E \approx E^{\prime}$ such that $W \mid K=$ identity and $\Phi^{\prime} W=\Phi$. Define $d^{\prime}=W d$. Then $\Phi^{\prime} d^{\prime}=\bar{\phi}^{\prime} W d=\bar{\phi} d=u ; \mu \bar{\phi}^{\prime} d^{\prime}=\mu u=1 ; \operatorname{dev} d^{\prime}=\operatorname{dev}(W d)=W \operatorname{dev} d=W f$ $=f$ Q.E.D.

Lemma (7.9). Let $(f, u)$ and $\left(f^{\prime}, u^{\prime}\right)$ be structural cocycles in $(\phi, \theta)$. Let $(E, \bar{\phi})$ and $\left(E^{\prime}, \bar{\phi}^{\prime}\right)$ be extensions of $(\phi, \theta)$. Consider the propositions

(A) $(f, u)$ represents $(E, \bar{\phi})$,

(B) $\left(f^{\prime}, u^{\prime}\right)$ represents $\left(E^{\prime}, \bar{\phi}^{\prime}\right)$,

(C) $(f, u)$ is equivalent to $\left(f^{\prime}, u^{\prime}\right)$,

(D) $(E, \bar{\phi})$ is isomorphic to $\left(E^{\prime}, \bar{\phi}^{\prime}\right)$.

We assert that any three of these together imply the fourth.

Proof. First suppose (A), (B), (C) hold. Choose right inverses $d^{\prime}$ and $d$ of $\mu \bar{\phi}^{\prime}: E^{\prime} \rightarrow Q$ and $\mu \bar{\phi}: E \rightarrow Q$, respectively, satisfying (7.2) with and without primes, respectively; choose a 1-cochain $h$ on $Q$ in $K$ satisfying (7.7). Transform the notation of Lemma 5.24 as follows: ( $f, t, Q, K, E, \rho, d, f^{\prime}, t^{\prime}, Q^{\prime}, K^{\prime}$, $\left.E^{\prime}, \rho^{\prime}, d^{\prime}, \phi, \theta, r, \bar{\phi}\right) \rightarrow\left(f^{\prime}, \theta u^{\prime}, Q, K, E^{\prime}, \mu \bar{\phi}^{\prime}, d^{\prime}, f, \theta u, Q, K, E, \mu \bar{\phi}, d, 1,1, h, W\right)$. Requirement (5.26a) then becomes (7.7a); requirement (5.26b) becomes $\theta u^{\prime}=c h+\theta u$, which follows from (7.7b). Therefore, by Lemma 5.24, there exists one and only one homorphism $W: E^{\prime} \rightarrow E$ satisfying (1.6a) and

$$
W d^{\prime}=h+d \text {. }
$$

It is easily verified that $W$ is $1-1$ onto; its inverse $W^{-1}: E \rightarrow E^{\prime}$ is characterized by $W^{-1} \mid K=$ identity and

$$
W^{-1} d=-h+d^{\prime}
$$

$\Phi W|K=\bar{\phi}| K=\phi=\bar{\phi}^{\prime} \mid K ; \quad \Phi W d^{\prime}=\bar{\phi} h+\bar{\phi} d=\phi h+u=u^{\prime}=\bar{\phi}^{\prime} d^{\prime}, \quad$ hence $\Phi W\left|d^{\prime}(Q)=\Phi^{\prime}\right| d^{\prime}(Q)$. It follows that $\Phi W=\Phi^{\prime}$, completing the proof of (D).

Next suppose (A), (B), (D) hold. It follows from (B), (D), and Lemma 7.8 that $\left(f^{\prime}, u^{\prime}\right)$ represents $(E, \Phi)$. This, (A), and Lemma 7.6 imply $(\mathrm{C})$.

Finally $\left({ }^{12}\right)$, suppose (A), (C), and (D) hold. By Lemma (7.4), $\left(f^{\prime}, u^{\prime}\right)$ represents some extension $\left(E^{\prime \prime}, \Phi^{\prime \prime}\right)$. By the established fact that $(\mathrm{A}),(\mathrm{B})$, (C) together imply $(\mathrm{D}),(E, \bar{\phi})$ is isomorphic to $\left(E^{\prime \prime}, \bar{\phi}^{\prime \prime}\right)$. Hence, by (D), $\left(E^{\prime \prime}, \Phi^{\prime \prime}\right)$ is isomorphic to $\left(E^{\prime}, \Phi^{\prime}\right)$. Therefore by Lemma $7.8,\left(f^{\prime}, u^{\prime}\right)$ represents $\left(E^{\prime}, \Phi^{\prime}\right)$. Q.E.D.

Combining Lemmas 7.1, 7.4, and 7.9, we obtain

THEOREM (7.12). The relation “ $(f, u)$ represents $(E, \bar{\phi})$ " induces a 1-1

(12) The assertion that (B), (C), (D) imply (A) is the same as that (A), (C), (D) imply (B), except for a change of notation. In any case, only the two parts of the lemma which have already been proved are needed for Theorem 7.12; but the third ( $=$ fourth) part is technically useful, particularly in the special case $(E, \bar{\phi})=\left(E^{\prime}, \bar{\phi}^{\prime}\right)$. 
correspondence between $\bar{H}^{2}(\phi, \theta)$ and the set of all isomorphism-classes of extensions of $(\phi, \theta)$.

8. Extensions of crossed modules: $H^{2}(Q, X)$. We continue the discussion of a fixed crossed module $(\phi, \theta), \theta: G \rightarrow A_{X}(K)$ maps $N$ onto $I(K)$ and hence induces a homomorphism $\theta_{\sharp}: Q \rightarrow A_{X}(K) / I(K)$, which is characterized by the relation $\theta_{*} \mu=\nu \theta$. Since $X \subset Z_{K}$, the natural homomorphism $\eta_{*}$ : $A_{X}(K) / I(K) \rightarrow A\left(X \cap Z_{K}\right)$ introduced in $\$ 2$ may be written $\eta_{*}: A_{X}(K) / I(K)$ $\rightarrow A(X)$. We consider the cohomology groups $H^{n}(Q, X)$ as defined relative to the operation $\eta_{*} \theta_{\sharp}: Q \rightarrow A(X)$ of $Q$ on $X$.

Lemma (8.1). If $u$ is a right inverse of $\mu: G \rightarrow Q$, then

$$
\nu \theta u=\theta_{\sharp}, \quad \eta \theta u=\eta_{*} \theta \dot{*} .
$$

Proof. $\nu \theta u=\theta_{\sharp} \mu u=\theta_{\sharp}$. Therefore $\eta \theta u=\eta_{*} \nu \theta u=\eta_{*} \theta_{*}$. Q.E.D.

Formula (8.2b) justifies various tacit applications of Lemmas 4.4 and 4.13.

Lemma (8.3). If $(f, u)$ is a structural cocycle in $(\phi, \theta)$, and $u^{\prime}$ is a right inverse of $\mu: G \rightarrow Q$, then there exists a 2-cochain $f^{\prime}$ on $Q$ in $K$ such that $\left(f^{\prime}, u^{\prime}\right)$ is a structural cocycle in $(\phi, \theta)$ equivalent to $(f, u)$.

Proof. Clearly $u^{\prime}-u \subset N$. Therefore we may choose a 1 -cochain $h$ on $Q$ in $K$ satisfying (7.7b). Define $f^{\prime}$ by (7.7a). Then it is easily verified by direct calculation that $\left(f^{\prime}, u^{\prime}\right)$ is a structural cocycle in $(\phi, \theta)$. By construction, $\left(f^{\prime}, u^{\prime}\right)$ is equivalent to $(f, u)$. Q.E.D.

Given any structural cocycle $(f, u)$ in $(\phi, \theta)$, and any $g \in Z^{2}(Q, X)$, we define

$$
g+(f, u)=(g+f, u) .
$$

$\phi(g+f)=\phi g+\phi f=\phi f=\operatorname{dev} u ; \delta_{\theta u} ;(g+f)=\delta g+\delta_{\theta u} f=0$. Therefore $g+(f, u)$ is a structural cocycle in $(\phi, \theta)$.

TheOREM (8.5). Formula (8.4) induces an operation of $H^{2}(Q, X)$ as a simply transitive transformation group on $\bar{H}^{2}(\phi, \theta)$, and hence, via Theorem 7.12 , on the set of all isomorphism-classes of extensions of $(\phi, \theta)$.

Proof. If $(f, u)$ is a structural cocycle, $[f, u]$ will denote the element of $\bar{H}^{2}(\phi, \theta)$ of which $(f, u)$ is a member. If $g \in Z^{2}(Q, X),[g]$ will denote the cohomology class containing $g$.

Given $g \in Z^{2}(Q, X)$ and $[f, u] \in \bar{H}^{2}(\phi, \theta)$, we define

$$
g+[f, u]=[g+f, u] \text {. }
$$

To show that this is independent of the choice of $(f, u)$, let $\left(f^{\prime}, u^{\prime}\right)$ be any structural cocycle equivalent to $(f, u)$. Choose a 1-cochain $h$ on $Q$ in $K$ satisfying (7.7). Using (4.8), we have $\left(g+f^{\prime}, u^{\prime}\right)=\left(g+h_{\theta u} \cdot f, \phi h+u\right)=\left(h_{\theta u} \cdot(g+f)\right.$, $\phi h+u)$ which is equivalent to $(g+f, u)$.

Clearly $g+\left(g^{\prime}+[f, u]\right)=\left(g+g^{\prime}\right)+[f, u]$, and $0+[f, u]=[f, u]$. Therefore 
we have defined an operation of $Z^{2}(Q, X)$ as a transformation group on $\bar{H}^{2}(\phi, \theta)$.

Let $[f, u]$ and $\left[f^{\prime}, u^{\prime}\right]$ be any two elements of $\bar{H}^{2}(\phi, \theta)$. By Lemma 8.3, there exists a 2-cochain $f^{\prime \prime}$ on $Q$ in $K$ such that $\left(f^{\prime \prime}, u^{\prime}\right)$ is a structural cocycle in $(\phi, \theta)$ equivalent to $(f, u)$. Let $g=f^{\prime}-f^{\prime \prime}$. Then $\phi g=\phi f^{\prime}-\phi f^{\prime \prime}=\operatorname{dev} u^{\prime}-\operatorname{dev} u^{\prime}=0$; therefore $g \subset X$. Furthermore $\delta g=\delta_{\theta u^{\prime}} f^{\prime}-\delta_{\theta u^{\prime}} f^{\prime \prime}=0$. Therefore $g \in Z^{2}(Q, X)$. Now $g+[f, u]=g+\left[f^{\prime \prime}, u^{\prime}\right]=\left[g+f^{\prime \prime}, u^{\prime}\right]=\left[f^{\prime}, u^{\prime}\right]$. Therefore $Z^{2}(Q, X)$ operates transitively on $\bar{H}^{2}(\phi, \theta)$.

Given $g \in B^{2}(Q, X)$, choose a 1-cochain $h$ on $Q$ in $X$ such that $\delta h=g$. Let $(f, u)$ be any structural cocycle in $(\phi, \theta)$. Then $(g+f, u)=(\delta h+f, u)$ $=\left(h_{\theta u} \cdot f, \phi h+u\right)$ which is equivalent to $(f, u)$. It follows that $B^{2}(Q, X)$ operates trivially on $\bar{H}^{2}(\phi, \theta)$. Consequently the operation of $Z^{2}(Q, X)$ on $\bar{H}^{2}(\phi, \theta)$ induces an operation of $H^{2}(Q, X)$ on $\bar{H}^{2}(\phi, \theta)$, by

$$
[g]+[f, u]=g+[f, u]=[g+f, u] ;
$$

the induced operation is still transitive.

Now suppose $[g] \in H^{2}(Q, X)$ and $[f, u] \in \bar{H}^{2}(\phi, \theta)$ are such that $[g]+[f, u]$ $=[f, u]$. This means that $(g+f, u)$ is equivalent to $(f, u)$. Choose a 1 -cochain $h$ on $Q$ in $K$ such that $g+f=h_{\theta u} \cdot f$ and $u=\phi h+u$. Then $\phi h=0$, and $h \subset X$; therefore $g+f=\delta h+f$; therefore $g=\delta h$; therefore $[g]=0$. This proves that the operation of $H^{2}(Q, X)$ on $\bar{H}^{2}(\phi, \theta)$ is simply transitive. Q.E.D.

CoRollary (8.8). If $(\phi, \theta)$ is extendible, there exists a 1-1 correspondence (not "natural," in general) between $H^{2}(Q, X)$ and the set of all isomorphismclasses of extensions of $(\phi, \theta)$.

9. The obstruction of a crossed module. The obstruction of a crossed module has been defined in [7], and before that was mentioned in [6]. Consequently this section, like $\$ 5$, is in the nature of a review, except for Corollary 9.5 , which was first given in [13] and has not previously been published.

A structural cochain in the crossed module $(\phi, \theta)$ is an ordered pair $(f, u)$, where $f$ is a 2-cochain on $Q$ in $K$, and $u$ is a right inverse of $\mu: G \rightarrow Q$, satisfying $\phi f=\operatorname{dev} u$ but not necessarily $\delta_{\theta u} f=0$. We define the coboundary $\delta(f, u)$ of a structural cochain $(f, u)$ by

$$
\delta(f, u)=\delta_{\theta u} f .
$$

We say that two structural cochains $(f, u)$ and $\left(f^{\prime}, u^{\prime}\right)$ are equivalent if there exists a 1 -cochain $h$ on $Q$ in $K$ satisfying (7.7).

If $u$ is any right inverse of $\mu: G \rightarrow Q$, then $\operatorname{dev} u \subset N$, and there exists a 2-cochain $f$ on $Q$ in $K$ such that $\phi f=\operatorname{dev} u$. Thus, although a pseudo-module need not admit a structural cocycle, every pseudo-module admits a structural cochain.

Lemma (9.2). If $(f, u)$ is a structural cochain in $(\phi, \theta)$, and $u^{\prime}$ is a right 
inverse of $\mu: G \rightarrow Q$, then there exists a 2-cochain $f^{\prime}$ on $Q$ in $K$ such that $\left(f^{\prime}, u^{\prime}\right)$ is a structural cochain equivalent to $(f, u)$.

The proof goes exactly as in Lemma 8.3, except of course that we do not conclude $\delta_{\theta u} f^{\prime}=0$.

Lemma (9.3). Any two equivalent structural cochains in $(\phi, \theta)$ have the same coboundary.

Proof. Let $(f, u)$ and $\left(f^{\prime}, u^{\prime}\right)$ be equivalent structural cochains; choose a 1-cochain $h$ on $Q$ in $K$ satisfying (7.7). Then $\theta u^{\prime}=\theta \phi h+\theta u=c_{K} h+\theta u$; hence $(f, \theta u)$ and $\left(f^{\prime}, \theta u^{\prime}\right)$ are equivalent structural cochains in the $Q$-kernel $\theta_{f}: Q$ $\rightarrow A_{X}(K) I(K)$. Therefore by Lemma 5.16, $\delta_{\theta u} f=\delta_{\theta u} f^{\prime}$. Q.E.D.

THEOREM (9.4). Let $\bar{C}^{2}(\phi, \theta)$ denote the set of all structural cochains in $(\phi, \theta)$. The mapping $\delta: \bar{C}^{2}(\phi, \theta) \rightarrow C^{3}(Q, K)$ defined by $(9.1)$ maps $\bar{C}^{2}(\phi, \theta)$ into, and onto, a single coset $\left({ }^{9}\right)$ of $B^{3}(Q, X)$ in $Z^{3}(Q, X)$.

We omit the proof, since the result has been established in [7].

The cohomology class $\delta\left[\bar{C}^{2}(\phi, \theta)\right]$, regarded as an element of $H^{3}(Q, X)$, is called the obstruction of $(\phi, \theta)$.

By Corollary 7.5, $(\phi, \theta)$ is extendible if and only if it admits a structural cocycle. But by Theorem 9.4 and the definition of the obstruction, $(\phi, \theta)$ admits a structural cocycle if and only if its obstruction vanishes. Hence

Corollary (9.5). A crossed module is extendible if and only if its obstruction vanishes.

10. Extensions of pseudo-modules. Let $(\phi, \theta)$ be an arbitrary pseudomodule; let $\left(\Gamma, \epsilon, \pi_{1}, \pi_{2}\right)$ be the graph of $\theta: G \rightarrow A_{X}(K) / c(X)$ rel. $\lambda: A_{X}(K)$ $\rightarrow A_{X}(K) / c(X)$, as in $\S 6$, and let $N^{\prime}=\phi^{\prime}(K)=\pi_{1}^{-1}(N)$.

$\theta: G \rightarrow A_{X}(K) / c(X)$ induces a homomorphism $\theta_{\sharp}: Q \rightarrow A_{X}(K) / I(K)$, characterized by $\theta_{t} \mu=\omega \theta$. The operation of $Q$ on $X \cap Z_{K}$ which we should naturally consider is $\eta_{*} \theta_{\sharp}: Q \rightarrow A\left(X \cap Z_{K}\right)$. However, if we wish to combine Theorem 8.5 with Corollary 6.10 to obtain a classification of the extensions of $(\phi, \theta)$, we have to consider the operation $\eta_{*}\left(\pi_{2}\right)_{\sharp}: Q \rightarrow A\left(X \cap Z_{K}\right)$, where $\left(\pi_{2}\right)_{\sharp}: Q$ $\rightarrow A_{X}(K) / I(K)$ is defined by $\left(\pi_{2}\right)_{f} \mu \pi_{1}=\nu \pi_{2}$. We note that $\eta_{*}: A_{X}(K) / I(K)$ $\rightarrow A\left(X \cap Z_{K}\right)$ in the sense of $\S 2$ is the restriction to $A_{X}(K) / I(K)$ of the natural homomorphism $\eta_{*}: A_{X} \cap z_{K}(K) / I(K) \rightarrow A\left(X \cap Z_{K}\right)$ in the sense of $\S 8$, as applied to the crossed module $\left(\phi^{\prime}, \pi_{2}\right)$.

Lemma $(10.1) .\left(\pi_{2}\right)_{f}=\theta_{\# \text {. }}$.

Proof. $\theta_{\sharp \mu} \pi_{1}=\omega \theta \pi_{1}=\omega \lambda \pi_{2}=\nu \pi_{2}=\left(\pi_{2}\right)_{\sharp \mu} \pi_{1}$. Since $Q=\mu \pi_{1}(\Gamma)$, this proves the lemma.

Consequently there is no ambiguity in the definition of the cohomology groups $H^{n}\left(Q, X \cap Z_{K}\right)$. Combining Theorem 8.5 with Corollary 6.10, we obtain 
THEOREM (10.2). $H^{2}\left(Q, X \cap Z_{K}\right)$ operates in a natural way as a simply transitive transformation group on the set of all isomorphism-classes of extensions of $(\phi, \theta)$.

We define the obstruction of the pseudo-module $(\phi, \theta)$ to be the obstruction of the crossed module $\left(\phi^{\prime}, \pi_{2}\right)$; it is an element of $H^{3}\left(Q, X \cap Z_{K}\right)$. Combining Corollary 9.5 with Corollary 6.11, we have

Corollary (10.3). A pseudo-module is extendible if and only if its obstruction vanishes.

With Theorem 10.2 and Corollary 10.3 our main objectives have been accomplished. The remainder of this section is devoted to machinery, with the object of casting the results on extensions of pseudo-modules in more explicit form.

We naturally define a structural cochain in $(\phi, \theta)$ as a structural cochain in the crossed module $\left(\phi^{\prime}, \pi_{2}\right)$. We say that a structural cocycle in $(\phi, \theta)$ represents an extension of $(\phi, \theta)$ if it represents the corresponding $\left({ }^{13}\right)$ extension of $\left(\phi^{\prime}, \pi_{2}\right)$.

If we regard a single map $u$ into $\Gamma$ as a pair of maps $(v, t)$ into $G$ and $A_{X}(K)$, respectively, satisfying the condition $\theta v=\lambda t$, then a structural cochain in $(\phi, \theta)$ can be regarded as an ordered triple $(f, v, t)$, where $f$ is a 2-cochain on $Q$ in $K, v$ is a right inverse of $\mu: G \rightarrow Q$, and $t$ is a 1-cochain on $Q$ in $A_{X}(K)$, satisfying the conditions

$$
\theta v=\lambda t, \quad \phi f=\operatorname{dev} v, \quad c_{K} f=\operatorname{dev} t .
$$

In this sense, the coboundary of a structural cochain $(f, v, t)$ is given by

$$
\delta(f, v, t)=\delta_{t} f
$$

Two structural cochains $(f, v, t)$ and $\left(f^{\prime}, v^{\prime}, t^{\prime}\right)$ are equivalent if and only if there exists a 1-cochain $h$ on $Q$ in $K$ satisfying

$$
f^{\prime}=h_{t} \cdot f, \quad v^{\prime}=\phi h+v, \quad t^{\prime}=c h+t .
$$

A structural cocycle $(f, v, t)$ represents an extension $(E, \bar{\phi})$, in the sense defined above, if and only if there exists a right inverse $d$ of $\mu \bar{\phi}: E \rightarrow Q$ satisfying

$$
f=\operatorname{dev} d, \quad v=\phi d, \quad t=C_{K} d .
$$

If we take formulae (10.4)-(10.7) as the definitions of "structural cochain," "coboundary," "equivalent," and "represents," the formal analogues of the propositions of $\$ \S 7-9$ all go through, with $X$ replaced by $X \cap Z_{K}$; this assertion follows from what we have already proved. The obstruction of the pseudo-module $(\phi, \theta)$ is the set of all coboundaries of all structural cochains

(13) Via Theorem 6.7. 
in $(\phi, \theta)$, in the sense of formulae (10.4) and (10.5). The operation of $H^{2}\left(Q, X \cap Z_{K}\right)$ on the set $\bar{H}^{2}(\phi, \theta)$ of all equivalence classes of structural cocycles in $(\phi, \theta)$ is induced by the formula

$$
g+(f, v, t)=(g+f, v, t)
$$

for $g \in Z^{2}\left(Q, X \cap Z_{K}\right)$, and $(f, v, t)$ a structural cocycle in $(\phi, \theta)$.

11. Frames. Throughout this section and the next we shall consider a fixed normal homomorphism $\phi: K \rightarrow G$.

Let $\theta: G \rightarrow A_{X}(K) / c(X)$ be a modular structure on $\phi: K \rightarrow G$; let $u$ be a right inverse of $\mu: G \rightarrow Q$. Define

$$
s=\theta u .
$$

Then the following are easily verified:

$$
\phi_{*} s=C_{N} u, \quad \operatorname{dev} s=\psi \operatorname{dev} u .
$$

Let $\theta: G \rightarrow A_{X}(K) / c(X)$ be a fixed modular structure, and let $u$ and $u^{\prime}$ be right inverses of $\mu: G \rightarrow Q$. Define $s=\theta u, s^{\prime}=\theta u^{\prime}$. Then

$$
s^{\prime}-s=\psi\left(u^{\prime}-u\right) \text {. }
$$

A frame in $\phi: K \rightarrow G$ is an ordered pair $(u, s)$, where $u$ is a right inverse of $\mu: G \rightarrow Q$, and $s$ is a 1 -cochain on $Q$ in $A_{X}(K) / c(X)$, satisfying (11.2). Two frames $(u, s)$ and $\left(u^{\prime}, s^{\prime}\right)$ are equivalent if they satisfy (11.3); this relation is reflexive, symmetric, and transitive.

Let $(u, s)$ be a frame in $\phi: K \rightarrow G$, and $\theta: G \rightarrow A_{X}(K) / c(X)$ a modular structure on $\phi: K \rightarrow G$. We say that $(u, s)$ is associated with $\theta$ if (11.1) holds.

THEOREM (11.4). The relation of "association" induces a 1-1 correspondence between the set of all modular structures on $\phi: K \rightarrow G$ and the set of all equivalence classes of frames in $\phi: K \rightarrow G$. That is: every modular structure is associated with at least one frame; every frame is associated with one and only one modular structure; two frames are associated with the same modular structure if and only if they are equivalent.

Proof. We have already indicated that every modular structure is associated with at least one frame.

Transform the notation of Lemma 5.19 as follows: $\left(E, \rho, Q, K, E^{\prime}, \phi, d, v, \bar{\phi}\right)$ $\rightarrow\left(G, \mu, Q, N, A_{X}(K) / c(X), \psi, u, s, \theta\right)$. Then conditions (5.20) become (3.5) and (11.1). Conditions (5.21) become (11.2b) and $\psi\left[\left(C_{N} u q\right) n\right]=s(q)+\psi(n)$ $-s(q)$. But by (2.15), $s(q)+\psi(n)-s(q)=\psi\left[\left(\phi_{*} s q\right) n\right]$. Hence $(5.21 \mathrm{~b})$ may be written $\psi\left[\left(C_{N} u q\right) n\right]=\psi\left[\left(\phi_{*} s q\right) n\right]$, which is implied by (11.2a). Now it is easily seen that if $\theta: G \rightarrow A_{X}(K) / c(X)$ is a homomorphism, then conditions (3.5), (11.1), and (11.2a) together imply commutativity throughout (3.4). It therefore follows from Lemma 5.19 that every frame is associated with one and only one modular structure. The last part of the theorem follows from Lemma 5.22. Q.E.D. 
12. Classification of continuations: $\bar{H}^{2}(\phi)$. We continue the discussion of a fixed normal homomorphism $\phi: K \rightarrow G$.

LEMMA (12.1). Let $f$ be a 2-cochain on $Q$ in $K$, $v$ a right inverse of $\mu: G \rightarrow Q$, and $t$ a 1-cochain on $Q$ in $A_{X}(K)$, satisfying (10.4b) and (10.4c). Then there exists at most one modular structure $\theta: G \rightarrow A_{X}(K) / c(X)$ on $\phi: K \rightarrow G$ such that $(f, v, t)$ is a structural cochain in the pseudo-module $(\phi, \theta)$. Moreover, there exists such a modular structure if and only if

$$
\phi_{\#} t=C_{N} v \text {. }
$$

Proof. If there exists such a modular structure $\theta$, then by $(10.4 \mathrm{a}), \phi_{\sharp t} t$ $=\phi_{*} \lambda t=\phi_{*} \theta v=C_{N} v$. Conversely, if (12.2) holds, then $\phi_{*} \lambda t=C_{N} v$, and $\operatorname{dev}(\lambda t)$ $=\lambda \operatorname{dev} t=\lambda c f=\psi \phi f=\psi \operatorname{dev} v$; hence $(v, \lambda t)$ is a frame. Therefore, by Theorem 11.4 , there exists one and only one modular structure $\theta: G \rightarrow A_{X}(K) / c(X)$ such that (10.4) holds. Q.E.D.

Definition. A structural cochain in $\phi: K \rightarrow G$ is an ordered triple $(f, v, t)$, where $f$ is a 2-cochain on $Q$ in $K, v$ is a right inverse of $\mu: G \rightarrow Q$, and $t$ is a 1-cochain on $Q$ in $A_{X}(K)$, satisfying (10.4b), (10.4c), and (12.2). We define "coboundary" and "equivalence" of structural cochains by formulae (10.5) and (10.6). A structural cocycle $(f, v, t)$ in $\phi: K \rightarrow G$ represents a continuation $(E, \bar{\phi})$ of $\phi: K \rightarrow G$ if there exists a right inverse $d$ of $\mu \bar{\phi}: E \rightarrow Q$ satisfying (10.7).

The set of all equivalence classes of all structural cocycles in $\phi: K \rightarrow G$ will be denoted $\bar{H}^{2}(\phi)$. If two structural cocycles $(f, v, t)$ and $\left(f^{\prime}, v^{\prime}, t^{\prime}\right)$ are equivalent, then their frames $(v, \lambda t)$ and $\left(v^{\prime}, \lambda t^{\prime}\right)$ are equivalent, and hence $(f, v, t)$ and $\left(f^{\prime}, v^{\prime}, t^{\prime}\right)$ are structural cocycles in the same pseudo-module. Consequently $\bar{H}^{2}(\phi)$ is the disjoint union of all sets of the form $\bar{H}^{2}(\phi, \theta)$, where $\theta$ ranges through all modular structures on $\phi: K \rightarrow G$. Moreover, if a structural cocycle in $\phi: K \rightarrow G$ represents a continuation of $\phi: K \rightarrow G$, then the modular structure determined by the continuation is the same as the modular structure associated with the frame of the structural cocycle. Therefore

TheOREM (12.3). The relation " $(f, v, t)$ represents $(E, \Phi)$ " induces a 1-1 correspondence between $\bar{H}^{2}(\phi)$ and the set of all isomorphism-classes of continuations of $\phi: K \rightarrow G$.

In fact, Lemma 7.9 holds, with “ $(\phi, \theta)$ " replaced by " $\phi: K \rightarrow G$," and "extensions" replaced by "continuations;" the notation " $(f, u)$ " must be expanded to " $(f, v, t)$."

REMARK. Theorem 12.3 cannot be derived from the special case of itself in which $X \subset Z_{K}$-at least, not in the manner of $\$ 10$-because in the normal homomorphism $\phi^{\prime}: K \rightarrow \Gamma$ defined in $\$ 6$, the group $\Gamma$ depends on the given modular structure. In other words, we have no way of associating with a given normal homomorphism another one whose continuation theory is equivalent to that of the first, and which satisfies $X \subset Z_{K}$. Moreover, when we 
are dealing with a normal homomorphism in which $X \subset Z_{K}$, and wish to consider structural cochains irrespective of any particular modular structure, we must regard them as triples $(f, v, t)$; they cannot be abbreviated to $(f, v)$ [or " $(f, u)$ "] until a particular modular structure has been specified. A structural cochain in the sense of $\$ 7$ is a structural cochain in every modular structure on $\phi: K \rightarrow G\left(X \subset Z_{K}\right)$, and may even be a structural cocycle in several different ones.

13. Splitting extensions. Let $P$ and $M$ be arbitrary groups; let $h$ be a 1 -cochain on $P$ in $M$, and $t$ a 1 -cochain on $P$ in $A(M)$. We say that $h$ is a crossed homomorphism rel. $t$ if $h_{t} \cdot 0=0$; that is, if

$$
h\left(p+p^{\prime}\right)=h(p)+(t q)\left(h p^{\prime}\right)
$$

for all $p, p^{\prime} \in P$.

Let $M$ be a normal subgroup of $P$, and let $\mu: P \rightarrow P / M$ be the natural homomorphism. We say that $P$ splits over $M$ if $\mu$ admits a right inverse homomorphism.

A splitting continuation of a normal homomorphism $\phi: K \rightarrow G$ is a continuation $(E, \Phi)$ such that $E$ splits over $K$. Clearly this is the case if and only if $\mu \Phi$ admits a right inverse homomorphism. A splitting extension of a pseudomodule $(\phi, \theta)$ is an extension of $(\phi, \theta)$ which is a splitting continuation of $\phi: K \rightarrow G$.

The following is self-evident:

Lemma (13.2). An extension of a crossed module is a splitting extension if and only if it can be represented by a structural cocycle $(f, u)$ in which $f=0$.

Lemma 13.2 remains true under the following transformations: [crossed module, $(f, u)] \rightarrow[$ pseudo-module, $(f, v, t)] ;$ [crossed module, $u] \rightarrow[Q$-kernel, $t]$; [a crossed module, $u] \rightarrow[Q$ by $K, t]$; [extension, crossed module, $(f, u)]$ $\rightarrow$ [continuation, normal homomorphism, $(f, v, t)]$.

By a splitting cocycle in a crossed module $(\phi, \theta)$ we mean a right inverse homomorphism $u: Q \rightarrow G$ of $\mu: G \rightarrow Q$. Two splitting cocycles $u$ and $u^{\prime}$ are equivalent if there exists a crossed homomorphism $h: Q \rightarrow K$ rel. $\theta u$ such that $u^{\prime}=\phi h+u$. The splitting cocycle $u$ represents the splitting extension $(E, \bar{\phi})$ if there exists a right inverse homomorphism $d: Q \rightarrow E$ of $\mu \Phi: E \rightarrow Q$ such that $\Phi d=u$. The set of all equivalence classes of splitting cocycles in $(\phi, \theta)$ will be denoted $\bar{H}_{0}^{2}(\phi, \theta)$. The passage from $u$ to $(0, u)$ induces a 1-1 mapping of $\bar{H}_{0}^{2}(\phi, \theta)$ into $\bar{H}^{2}(\phi, \theta)$. From Theorem 7.12 , we obtain

THEOREM (13.3). The relation " $u$ represents $(E, \Phi)$ " induces a 1-1 correspondence between $\bar{H}_{0}^{2}(\phi, \theta)$ and the set of all isomorphism-classes of splitting extensions of $(\phi, \theta)$.

Corollary (13.4). A crossed module admits a splitting extension if and only if $G$ splits over $N$. 
If $K$ is abelian, and $H^{2}(Q, K)=0$ (with $Q$ operating on $K$ by $\theta_{f}$ ), then, by [4], every extension of the " $Q$-kernel" $\theta_{\sharp}: Q \rightarrow A(K)$ splits over $K$. Hence every extension of $(\phi, \theta)$ is a splitting extension. Combining this with Corollary 13.4, we have

Corollary (13.5). Let $(\phi, \theta)$ be a crossed module in which $K$ is abelian and $H^{2}(Q, K)=0$. Then $(\phi, \theta)$ is extendible if and only if $G$ splits over $N$.

A splitting cocycle in the normal homomorphism $\phi: K \rightarrow G$ is an ordered pair $(v, t)$, where $v: Q \rightarrow G$ is a right inverse homomorphism of $\mu: G \rightarrow Q$ and $t: Q \rightarrow A_{X}(K)$ is a homomorphism, satisfying (12.2). Two splitting cocycles $(v, t)$ and $\left(v^{\prime}, t^{\prime}\right)$ are equivalent if there exists a crossed homomorphism $h: Q \rightarrow K$ rel. $t$ satisfying (10.6b) and (10.6c). The splitting cocycle $(v, t)$ represents the continuation $(E, \bar{\phi})$ if there exists a right inverse homomorphism $d: Q \rightarrow E$ of $\mu \bar{\phi}: E \rightarrow Q$ satisfying (10.7b) and (10.7c). The set of all equivalence classes of all splitting cocycles in $\phi: K \rightarrow G$ is denoted $\bar{H}_{0}^{2}(\phi)$; the passage from $(v, t)$ to $(0, v, t)$ induces a 1-1 mapping of $\bar{H}_{0}^{2}(\phi)$ into $\bar{H}^{2}(\phi)$. A splitting cocycle $(v, t)$ belongs to a particular modular structure $\theta: G$ $\rightarrow A_{X}(K) / c(X)$ if (and only if) formula (10.4a) holds. The set of all equivalence classes of all splitting cocycles in the pseudo-module $(\phi, \theta)$ is denoted $\bar{H}_{0}^{2}(\phi, \theta)$; the natural imbedding of $\bar{H}_{0}^{2}(\phi)$ in $\bar{H}^{2}(\phi)$ carries $\bar{H}_{0}^{2}(\phi, \theta)$ into $\bar{H}^{2}(\phi, \theta)$. The analogue of Theorem 13.3 carries through. Corollary 13.4, however, does not apply verbatim to arbitrary pseudo-modules. Given a pseudo-module $(\phi, \theta)$, let the groups $\Gamma$ and $N^{\prime}$ be defined as in $\S 6$. Then $(\phi, \theta)$ admits a splitting extension if and only if $\Gamma$ splits over $N^{\prime}$.

A splitting cocycle on $Q$ in $K$ is simply an arbitrary homomorphism $t: Q \rightarrow A(K)$. Two splitting cocycles $t$ and $t^{\prime}$ are equivalent if there exists a crossed homomorphism $h: Q \rightarrow K$ rel. $t$ such that $t^{\prime}=c h+t$. The splitting cocycle $t$ represents the extension $(E, \rho)$ of $Q$ by $K$ if there exists a right inverse homomorphism $d: Q \rightarrow E$ of $\rho: E \rightarrow Q$ such that $C_{K} d=t$. Each splitting cocycle $t$ lies in the $Q$-kernel $\nu t: Q \rightarrow A(K) / I(K)$. The analogues of Theorem 13.3 go through; the analogue of Corollary 13.4 is that a $Q$-kernel $\theta: Q \rightarrow A(K) / I(K)$ admits a splitting extension if and only if $\theta$ can be "lifted" to a homomorphism of $Q$ into $A(K)$.

REMARK. In a crossed module $(\phi, \theta)$, if $u$ and $u^{\prime}$ are splitting cocycles, then there always exists a 1 -cochain $h$ on $Q$ in $K$ such that $u^{\prime}=\phi h+u$. Moreover, for every such $h, \phi h$ is a crossed homomorphism rel. $C_{N} u$; for $0=\operatorname{dev} u^{\prime}$ $=\operatorname{dev}(\phi h+u)=(\phi h)_{C_{N} u} \cdot(\operatorname{dev} u)=(\phi h)_{C_{N} u} \cdot 0$. However, this does not by any means imply that $h$ itself can be so chosen as to be a crossed homomorphism rel. $\theta u$. We show this by an example in a $Q$-kernel (see [6] on the equivalence between " $Q$-kernel" and "crossed module in which $X=Z_{K}$ "):

Let $K$ be the additive (non-abelian) group of order 8 generated by symbols $a$ and $b$ with the relations $4 a=2 b=2(b+a)=0$; let $Q$ be the additive group of order 2 generated by $q_{0}$. Define homomorphisms $t, t^{\prime}: Q \rightarrow A(K)$ by 
$t\left(q_{0}\right)=1, t^{\prime}\left(q_{0}\right)=c_{K}(a)$. Then $t$ and $t^{\prime}$ represent splitting extensions of the same "trivial" $Q$-kernel, with $\theta(Q)=0$, and $\left(t^{\prime}-t\right): Q \rightarrow I(K)$ is a crossed homomorphism rel. $C_{I} t$, where $C_{I}: A(K) \rightarrow A[I(K)]$ is the natural homomorphism. But $t^{\prime}$ and $t$ are not equivalent, and they represent non-isomorphic extensions. For if $h: Q \rightarrow K$ is a crossed homomorphism rel. $t$ such that $c h=t^{\prime}-t$, then $h\left(q_{0}\right)$ is an element of $K$ such that $2 h\left(q_{0}\right)=0$ and $c_{K}\left[h\left(q_{0}\right)\right]=c_{K}(a)$. It happens that no such element $h\left(q_{0}\right)$ of $K$ exists. $t$, of course, represents the "direct sum" extension $K+Q ; t^{\prime}$ does not.

14. Classification of pseudo-modules in terms of crossed modules. We shall call two pseudo-modules $(\phi, \theta)$ and $\left(\phi^{\prime}, \theta^{\prime}\right)$ strictly isomorphic [3] if $K=K^{\prime}$ and there exists an isomorphism $W: G \approx G^{\prime}$ satisfying

$$
W \phi=\phi^{\prime}, \quad \theta^{\prime} W=\theta .
$$

A modular pair is an ordered pair $(M, Y)$, where $M$ is a crossed module with all the usual notation, and $Y$ is a subgroup of $K$ satisfying

$$
Y \cap Z_{K}=X, \quad A_{Y}(K) \supset \theta(G) .
$$

It follows from (14.2b) that $Y$ is a normal subgroup of $K$.

Two modular pairs $(M, Y)$ and $\left(M^{\prime}, Y^{\prime}\right)$ are strictly isomorphic if $M$ and $M^{\prime}$ are strictly isomorphic, and $Y=Y^{\prime}$.

If $(\phi, \theta)$ is a pseudo-module, the crossed module $\left(\phi^{\prime}, \pi_{2}\right)$ constructed in $\$ 6$ will be called the crossed module associated with $(\phi, \theta)$. The modular pair $\left[\left(\phi^{\prime}, \pi_{2}\right), X\right]$ will be called the modular pair associated with $(\phi, \theta)$. Here, $X$ denotes $\phi^{-1}(0)$, not $\phi^{\prime-1}(0)$.

Let $[(\phi, \theta), Y]$ be a modular pair. From (14.2b) and the condition $\phi \sharp \theta=C_{N}$ it follows that $\phi(Y)$ is a normal subgroup of $G$. Define $G^{\prime}=G / \phi(Y)$, and let $\beta: G \rightarrow G^{\prime}$ denote the natural homomorphism. $\theta: G \rightarrow A_{Y}(K) \operatorname{maps} \phi(Y)$ onto $c_{K}(Y)$ and hence induces a homomorphism $\theta^{\prime}: G^{\prime} \rightarrow A_{Y}(K) / c(Y)$. It is easily verified that the homomorphisms $\beta \phi: K \rightarrow G^{\prime}, \theta^{\prime}: G^{\prime} \rightarrow A_{Y}(K) / c(Y)$ constitute a pseudo-module; $(\beta \phi)^{-1}(0)=Y$. The pseudo-module $\left(\beta \phi, \theta^{\prime}\right)$ will be called the pseudo-module associated with the modular pair $[(\phi, \theta), Y]$.

TheOREm (14.3). The concept of a pseudo-module is equivalent, via the above constructions, to the concept of a modular pair.

Proof. First let $(\phi, \theta)$ be a pseudo-module, with the usual notation, and let $\left[\left(\phi^{\prime}, \pi_{2}\right), X\right]$ be its associated modular pair, with the notation of $\$ 6$. Let $\beta: \Gamma \rightarrow \Gamma / \phi^{\prime}(X)$ denote the natural homomorphism. The homomorphism $\pi_{2}: \Gamma \rightarrow A_{X}(K)$ maps $\phi^{\prime}(X)$ onto $c(X)$ and therefore induces a homomorphism $\pi_{2 t}: \Gamma / \phi^{\prime}(X) \rightarrow A_{X}(K) / c(X)$, characterized by $\pi_{2 \sharp} \beta=\lambda \pi_{2}$. Then $\left(\beta \phi^{\prime}, \pi_{2 \sharp}\right)$ is the pseudo-module associated with the modular pair $\left[\left(\phi^{\prime}, \pi_{2}\right), X\right]$; we must prove that $\left(\beta \phi^{\prime}, \pi_{2 f}\right)$ is strictly isomorphic to $(\phi, \theta)$.

It is easily verified that the sequence

$$
0 \rightarrow X \cap Z_{K} \rightarrow X \stackrel{\phi^{\prime}}{\rightarrow} \Gamma \stackrel{\pi_{1}}{\rightarrow} G \rightarrow 0
$$


is exact. Hence $\pi_{1}: \Gamma \rightarrow G$ induces an isomorphism $\pi_{1 \neq}: \Gamma / \phi^{\prime}(X) \approx G$, characterized by $\pi_{1 \neq} \beta=\pi_{1}$. Then $\theta \pi_{1 \neq} \beta=\theta \pi_{1}=\lambda \pi_{2}=\pi_{2 f} \beta$. Therefore $\theta \pi_{1 f}=\pi_{2 \sharp}$. Moreover, $\pi_{1 *} \beta \phi^{\prime}=\pi_{1} \phi^{\prime}=\phi$. Thus $\pi_{1 f}$ is the desired strict isomorphism between the two pseudo-modules.

Conversely, let $[(\phi, \theta), Y]$ be a modular pair, and let $\left(\beta \phi, \theta^{\prime}\right)$ be its associated pseudo-module, with the notation introduced just before the statement of the theorem; we use all our standard notation for the given crossed module $(\phi, \theta)$. Let $\Gamma$ denote the subgroup of $G^{\prime}+A_{Y}(K)$ characterized by $\theta^{\prime} \pi_{1}=\lambda \pi_{2}$; define $\phi^{\prime}: K \rightarrow \Gamma$ by $\phi^{\prime}(k)=\left[\beta \phi(k), c_{K}(k)\right]$. Since $(\beta \phi)^{-1}(0)=Y$, the modular pair associated with $\left(\beta \phi, \theta^{\prime}\right)$ is precisely $\left[\left(\phi^{\prime}, \pi_{2}\right), Y\right]$. Therefore our task is to prove that the crossed modules $(\phi, \theta)$ and $\left(\phi^{\prime}, \pi_{2}\right)$ are strictly isomorphic.

Define $W: G \rightarrow \Gamma$ by $W(g)=[\beta(g), \theta(g)]$. Such an ordered pair lies in $\Gamma$ by the relation $\theta^{\prime} \beta=\lambda \theta$, which characterizes $\theta^{\prime}: G^{\prime} \rightarrow A_{Y}(K) / c(X)$. It is easily verified that $W$ is the desired strict isomorphism between the two crossed modules; the proof that $W(G)=\Gamma$ involves an argument similar to the proof of Lemma 6.4.

For a classification of crossed modules and pseudo-modules in terms of $Q$-kernels, see $[3 ; 10]$.

\section{REFERENCES}

1. M. Auslander, Compound group extensions IV, forthcoming.

2. R. Baer, Erweiterung von Gruppen und ihren Isomorphismen, Math. Zeit. vol. 38 (1934) pp. 375-416.

3. A. P. Cobbe, Some algebraic properties of crossed modules, Quart. J. Math. Oxford Ser. (2) vol. 2 (1951) pp. 269-285.

4. S. Eilenberg and S. MacLane, Cohomology theory in abstract groups I, Ann. of Math. vol. 48 (1947) pp. 51-78. 341.

5. - Cohomology theory in abstract groups II, Ann. of Math. vol. 48 (1947) pp. 326736-761.

6. S. MacLane, Cohomology theory in abstract groups III, Ann. of Math. vol. 50 (1949) pp.

7. S. MacLane and J. H. C. Whitehead, On the 3-type of a complex, Proc. Nat. Acad. Sci. U.S.A. vol. 36 (1950) pp. 41-48.

8. O. Schreier, Über die Erweiterung von Gruppen, Monatshefte für Mathematik und Physik vol. 34 (1926) pp. 165-180.

9. R. L. Taylor, Compound group extensions II, forthcoming (Trans. Amer. Math. Soc.)

10. - Compound group extensions III, forthcoming.

11. - Covering groups of non-connected topological groups, forthcoming.

12. - Covering spaces, covering groups, and groupoids, forthcoming.

13. - On the classification of fibre bundles, Thesis, Oxford, 1950.

14. J. H. C. Whitehead, Combinatorial homotopy. II, Bull. Amer. Math. Soc. vol. 55 (1949) pp. $453-496$.

Columbia University, NEW YORK, N. Y. 